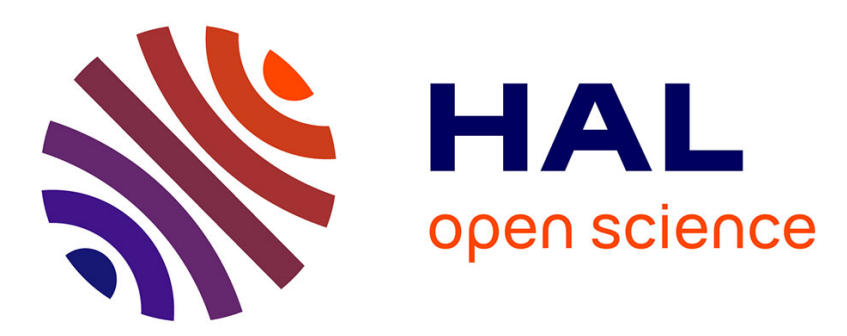

\title{
On Classical Solutions to the Mean Field Game System of Controls
}

\author{
Z Kobeissi
}

\section{To cite this version:}

Z Kobeissi. On Classical Solutions to the Mean Field Game System of Controls. 2020. hal02109214v2

\section{HAL Id: hal-02109214 \\ https://hal.science/hal-02109214v2}

Preprint submitted on 9 Jul 2020

HAL is a multi-disciplinary open access archive for the deposit and dissemination of scientific research documents, whether they are published or not. The documents may come from teaching and research institutions in France or abroad, or from public or private research centers.
L'archive ouverte pluridisciplinaire HAL, est destinée au dépôt et à la diffusion de documents scientifiques de niveau recherche, publiés ou non, émanant des établissements d'enseignement et de recherche français ou étrangers, des laboratoires publics ou privés. 


\title{
On Classical Solutions to the Mean Field Game System of Controls
}

\author{
Z. Kobeissi*
}

July 9, 2020

\begin{abstract}
We consider a class of mean field games in which the optimal strategy of a representative agent depends on the statistical distribution of the states and controls.

We prove some existence results for the forward-backward system of PDEs under rather natural assumptions. The main step of the proof consists of obtaining a priori estimates on the gradient of the value function by Bernstein's method. Uniqueness is also proved under more restrictive assumptions.

Finally, we discuss some examples to which the previously mentioned existence (and possibly uniqueness) results apply.
\end{abstract}

\section{1 - INTRODUCTION}

The theory of Mean Field Games (MFG for short) has been introduced in the independent works of J.M. Lasry and P.L. Lions [31, 32, 33], and of M.Y. Huang, P.E. Caines and R.Malhamé [25, 26]. It aims at studying deterministic or stochastic differential games (Nash equilibria) as the number of agents tends to infinity. The agents are supposed to be rational (given a cost to be minimized, they always choose the optimal strategies), and indistinguishable. Furthermore, the agents interact via some empirical averages of quantities which depend on the state variable.

At the limit when $N \rightarrow+\infty$, the game may be modeled by a system of two coupled partial differential equations (PDEs), which is named the MFG system. On the one hand, there is a Fokker-Planck-Kolmogorov equation describing the evolution of the statistical distribution $m$ of the state variable; this equation is a forward in time parabolic equation, and the initial distribution at time $t=0$ is given. On the other hand, the optimal value of a generic agent at some time $t$ and state $x$ is noted $u(t, x)$ and is defined as the lowest cost that a representative agent can achieve from time $t$ to $T$ if it is at state $x$ at time $t$. The value function satisfies a Hamilton-Jacobi-Bellman equation posed backward in time with a terminal condition involving a terminal cost. In the present work, we will restrict our attention to the case when the costs and the dynamics are periodic in the state variable, and we will work in the $d$-dimensional torus $\mathbb{T}^{d}$ (as it is often done in the MFG literature for simplicity). We will take a finite horizon time $T>0$, and will only consider second-order non-degenerate MFG systems. In this case, the MFG system is often written as:

$$
\begin{aligned}
& -\partial_{t} u(t, x)-\nu \Delta u(t, x)+H\left(t, x, \nabla_{x} u(t, x)\right)=f(x, m(t)) \\
& \partial_{t} m(t, x)-\nu \Delta m(t, x)-\operatorname{div}\left(H_{p}\left(t, x, \nabla_{x} u(t, x)\right) m\right)=0 \\
& u(T, x)=g(x, m(T)) \\
& m(0, x)=m_{0}(x)
\end{aligned}
$$

$$
\begin{array}{r}
\text { in }(0, T) \times \mathbb{T}^{d}, \\
\text { in }(0, T) \times \mathbb{T}^{d}, \\
\text { in } \mathbb{T}^{d}, \\
\text { in } \mathbb{T}^{d}
\end{array}
$$

${ }^{*}$ Laboratoire Jacques-Louis Lions, Univ. Paris Diderot, Sorbonne Paris Cité, UMR 7598, UPMC, CNRS, 75205, Paris, France. zkobeissi@math.univ-paris-diderot.fr 
We refer the reader to [10] for some theoretical results on the convergence of the $N$-agents Nash equilibrium to the solutions of the MFG system. For a thorough study of the well-posedness of the MFG system, see the videos of P.L.Lions' lecture at the Collège de France, and some lecture notes [9].

There is also an important literature on the probabilistic aspects of MFGs, see [12, 29] for some examples and [13, 14] for a detailed presentation of the probabilistic viewpoint.

For applications of MFGs, numerical simulations are crucial because it is most often impossible to find explicit or semi-explicit solutions to the MFG system. We refer to [2] for a survey on finite difference methods and to [3] for applications to crowd motion.

Most of the literature on MFGs is focused on the case when the mean field interactions only involves the distributions of states. Here we will consider a more general situation in which the cost of an individual agent depends on the joint distribution $\mu$ of states and optimal strategies. To underline this, we choose to use the terminology Mean Field Games of Controls (MFGCs) for this class of MFGs; the latter terminology was introduced in [11. Within this framework, the usual MFG system (1.1) is replaced by the following MFGC system,

$$
\begin{aligned}
& -\partial_{t} u(t, x)-\nu \Delta u(t, x)+H\left(x, \nabla_{x} u(t, x), \mu(t)\right)=0 \\
& \partial_{t} m(t, x)-\nu \Delta m(t, x)-\operatorname{div}\left(H_{p}\left(x, \nabla_{x} u(t, x), \mu(t)\right) m\right)=0 \\
& \mu(t)=\left(I_{d},-H_{p}\left(\cdot, \nabla_{x} u(t, \cdot), \mu(t)\right)\right) \# m(t) \\
& u(T, x)=g(x, m(T)) \\
& m(0, x)=m_{0}(x)
\end{aligned}
$$

$$
\begin{array}{r}
\text { in }(0, T) \times \mathbb{T}^{d}, \\
\text { in }(0, T) \times \mathbb{T}^{d}, \\
\text { in }[0, T], \\
\text { in } \mathbb{T}^{d}, \\
\text { in } \mathbb{T}^{d} .
\end{array}
$$

We would like to point out two of the main difficulties that one may encounter when studying (1.2) and which are not present in the study of (1.1).

1) The joint law of states and controls satisfies a fixed point relation described by (1.2c).

2) The HJB equation (1.2a) is non-local with respect to $\nabla_{x} u$. Consequently, it is much more difficult to obtain uniform a priori estimates on $u$ and the its derivatives.

Difficulty 1) is in general not straightforward and one needs to make assumptions for the fixed point in $\mu$ to have a unique solution when $\left(\nabla_{x} u, m\right)$ are given. An example in which this fixed point relation does not admit any solution is given in [1] Remark 4.3.

Let us provide a simple illustration for describing difficulty 2) by comparing the results obtained when we apply the maximum principle on parabolic equations to (1.1a) and (1.2a) respectively: if $u$ satisfies (1.1a) where $f$ and $g$ are assumed to be uniformly bounded with respect to $m$, then $u$ is uniformly bounded; under the same assumption on $g$, if $u$ is a solution to (1.2a) and $H$ is not uniformly bounded with respect to $\mu$, we can only say that $u$ is bounded in absolute value by a constant depending on $\mu$. The other estimates used in the usual arguments of existence in MFG sytems suffer the same lack of uniformity with respect to $\mu$. Conversely, the estimates of $\mu$ depend on $\nabla_{x} u$. It is not obvious a priori how to combine the estimates on $\mu$ and $(u, m)$ in order to obtain uniform estimates on $u$. Consequently, compactness results are harder to obtain for (1.2) than for (1.1).

The main assumption of this paper, namely FP1 and FP2 described below, is an original structural assumption designed to address difficulty 1). In particular, it implies that the map

$$
\mu \mapsto \widetilde{\mu}=\left(I_{d},-H_{p}\left(\cdot, \nabla_{x} u(t, \cdot), \mu\right)\right) \# m,
$$

is a contraction in a convenient metric space, when $(t, u, m)$ are given.

Moreover, we also assume that the Hamiltonian $H(x, p, \mu)$ behaves like a power function when $p$ tends to infinity. See paragraph 2.2 for more details. 
The main objective of this work is to discuss existence of the solutions of the MFGC system (1.2) within this framework. We will also give a uniqueness result under a short time horizon assumption. We refer to [1] for a numerical application with multiple solutions. Indeed, uniqueness does not hold in general for arbitrary time horizon. It can be obtained though, under a monotonicity assumption which is investigated in the companion paper [28]. In [28], existence and uniqueness of solutions of the MFGC system are proved under the above-mentioned monotonicity assumption and with Hamitonian having similar growth as in the present paper. This monotonicity condition implies that the agents favor moving in a direction opposite to the mainstream. Such an assumption is adapted to some models coming from finance or economy; and may be unrealistic in several situations, in particular in models of crowd motions. This explains why here we introduce a new structural assumption and refrain from assuming monotonicity or investigating uniqueness in the general case.

\section{Related literature}

In the first articles devoted to MFGCs, 20, 21, D. Gomes and his collaborators have given several existence results for MFGCs in various cases, using the terminology extended MFGs instead of MFGCs. For instance, [21] contains existence results for stationary games (infinite horizon) under the assumption that some of the parameters involved in the models are small. We refer to [7, 11, 15, 13, 28, for other existence and uniqueness results for MFGC systems.

Uniqueness is a major issue in MFG theory, it has been proved for (1.1) in [33, 35] under an assumptions called the Lasry-Lions monotonicity on the coupling function $f$ and the terminal cost $g$ in the case of non-local coupling. This assumption has been extended to MFGC and discussed in [20, 13, 28] in which uniqueness is proved. It translates the fact that the agents prefer directions opposite to the mainstream direction; therefore it is not adapted to a large class of MFGC systems like crowd motion models in which an agent is more likely to go in the mainstream direction.

The latter example of population dynamic is the typical application we had in mind when writing the assumptions in the present paper, see paragraphs 6.3 and 6.4. To our knowledge, existence results for such MFGC systems have not been discussed in the literature before. Uniqueness should not hold in general but under a short-time assumption. We refer to [1] in which the MFGC system is discretized using a finite-difference scheme and simulations are provided where the approximating discrete MFGC system admits several different solutions.

For other applications of MFGCs we refer to [11] for an model of optimal trading, [8, 16, 22, 24, 27] in the case of competition between firms producing the same goods, or [4] for energy storage.

\section{Organization of the paper}

Section 2 describes the notations, assumptions and main results in this paper. In Section 3 , we address difficulty 1) which consists of inverting the fixed point relation in $\mu(1.2 \mathrm{C})$ and providing estimates on the resulting flow of measures. Section 4 is devoted to proving a priori estimates on the solutions to (1.2) and addresses difficulty 2). Section 5 contains the proofs of the main results. Finally, we discuss several applications in Section 6. Namely, we study

- the Bertrand and Cournot competition for exhaustible ressources and introduce an extension to negatively correlated ressources (for instance gold and other raw materials);

- a model of price impact for high-frequency trading by Almgren and Chriss in which we discuss the possibility for the bid and ask prices to be different;

- a first-order flocking model;

- a crowd motion model. 


\section{2 - NOTATIONS AND ASSUMPTIONS}

\subsection{Notations and definitions}

The spaces of probability measures are equipped with the weak* topology. We denote by $\mathcal{P}_{\infty}\left(\mathbb{T}^{d} \times \mathbb{R}^{d}\right)$ the subset of measures $\mu$ in $\mathcal{P}\left(\mathbb{T}^{d} \times \mathbb{R}^{d}\right)$ with a second marginal compactly supported. For $\mu \in \mathcal{P}_{\infty}\left(\mathbb{R}^{d} \times \mathbb{R}^{d}\right)$ and $\widetilde{q} \in[1, \infty)$, we define the quantities $\Lambda_{\widetilde{q}}(\mu)$ and $\Lambda_{\infty}(\mu)$ by,

$$
\begin{aligned}
\Lambda_{\widetilde{q}}(\mu) & =\left(\int_{\mathbb{R}^{d} \times \mathbb{R}^{d}}|\alpha|^{\widetilde{q}} d \mu(x, \alpha)\right)^{\frac{1}{\widetilde{q}}}, \\
\Lambda_{\infty}(\mu) & =\sup \{|\alpha|,(x, \alpha) \in \operatorname{supp} \mu\} .
\end{aligned}
$$

Jensen inequality states that,

$$
\Lambda_{q_{1}}(\mu) \leq \Lambda_{q_{2}}(\mu)
$$

for any $1 \leq q_{1} \leq q_{2} \leq \infty$.

For $R>0$, we denote by $\mathcal{P}_{\infty, R}\left(\mathbb{R}^{d} \times \mathbb{R}^{d}\right)$ the subset of measures $\mu$ in $\mathcal{P}_{\infty}\left(\mathbb{R}^{d} \times \mathbb{R}^{d}\right)$ such that $\Lambda_{\infty}(\mu) \leq R$. The probability measures $\mu$ involved in (1.2) and (2.4), have a particular form, since they are the images of a measure $m$ on $\mathbb{T}^{d}$ by $\left(I_{d}, \alpha\right)$, where $\alpha$ is a bounded measurable functions from $\mathbb{T}^{d}$ to $\mathbb{R}^{d}$; in particular they are supported on the graph of $\alpha$. For $m \in \mathcal{P}\left(\mathbb{T}^{d}\right)$, we call $\mathcal{P}_{m}\left(\mathbb{T}^{d} \times \mathbb{R}^{d}\right)$ the set of such measures. For $\mu \in \mathcal{P}_{m}\left(\mathbb{T}^{d} \times \mathbb{R}^{d}\right)$, we set $\alpha^{\mu}$ to be the unique element of $L^{\infty}(m)$ such that $\mu=\left(I_{d}, \alpha^{\mu}\right) \# m$. Here, $\Lambda_{\widetilde{q}}(\mu)$ and $\Lambda_{\infty}(\mu)$ defined in (2.1) are given by

$$
\begin{aligned}
\Lambda_{\widetilde{q}}(\mu) & =\left\|\alpha^{\mu}\right\|_{L^{\widetilde{q}}(m)}, \\
\Lambda_{\infty}(\mu) & =\left\|\alpha^{\mu}\right\|_{L^{\infty}(m)} .
\end{aligned}
$$

If $X$ is a normed space and $|\cdot|_{X}$ is its norm, for $n \geq 1$ we denote by $C^{0}\left(X ; \mathbb{R}^{n}\right)$ the set of bounded continuous functions from $X$ to $\mathbb{R}^{n}$; it is endowed with the norm $\|v\|_{\infty}=$ $\sup _{x \in X}|v(x)|_{X}$.

We define $C^{0,1}\left([0, T] \times \mathbb{T}^{d} ; \mathbb{R}\right)$ as the set of the functions $v \in C^{0}\left([0, T] \times \mathbb{T}^{d} ; \mathbb{R}\right)$ differentiable at any point with respect to the state variable, and such that its gradient satisfies $\nabla_{x} v \in$ $C^{0}\left([0, T] \times \mathbb{T}^{d} ; \mathbb{R}^{d}\right)$. This is a Banach space equipped with the norm $\|v\|_{C^{0,1}}=\|v\|_{\infty}+\left\|\nabla_{x} v\right\|_{\infty}$.

For $\beta \in(0,1)$ and $n \geq 1$, we denote by $C^{\frac{\beta}{2}, \beta}\left([0, T] \times \mathbb{T}^{d} ; \mathbb{R}^{n}\right)$ the parabolic space of Hölder continuous functions which is commonly defined by

$C^{\frac{\beta}{2}, \beta}\left([0, T] \times \mathbb{T}^{d} ; \mathbb{R}^{n}\right)=\left\{\begin{array}{l}v \in C^{0}\left([0, T] \times \mathbb{T}^{d} ; \mathbb{R}^{n}\right), \exists C>0 \text { s.t. } \forall\left(t_{1}, x_{1}\right),\left(t_{2}, x_{2}\right) \in[0, T] \times \mathbb{T}^{d}, \\ \left|v\left(t_{1}, x_{1}\right)-v\left(t_{2}, x_{2}\right)\right| \leq C\left(\left|x_{1}-x_{2}\right|^{2}+\left|t_{1}-t_{2}\right|\right)^{\frac{\beta}{2}}\end{array}\right\}$

This is a Banach space equipped with the norm,

$$
\|v\|_{C^{\frac{\beta}{2}, \beta}}=\|v\|_{\infty}+\sup _{\left(t_{1}, x_{1}\right) \neq\left(t_{2}, x_{2}\right)} \frac{\left|v\left(t_{1}, x_{1}\right)-v\left(t_{2}, x_{2}\right)\right|}{\left(\left|x_{1}-x_{2}\right|^{2}+\left|t_{1}-t_{2}\right|\right)^{\frac{\beta}{2}}} .
$$

The space $C^{\frac{1+\beta}{2}, 1+\beta}\left([0, T] \times \mathbb{T}^{d} ; \mathbb{R}\right)$ is defined as the set of the functions $v \in C^{0,1}\left([0, T] \times \mathbb{T}^{d} ; \mathbb{R}\right)$ such that $\nabla_{x} v \in C^{\frac{\beta}{2}, \beta}\left([0, T] \times \mathbb{T}^{d} ; \mathbb{R}^{n}\right)$ and which admits a finite norm defined by,

$$
\|v\|_{C^{\frac{1+\beta}{2}, 1+\beta}}=\|v\|_{\infty}+\left\|\nabla_{x} v\right\|_{C^{\frac{\beta}{2}, \beta}}+\sup _{\left(t_{1}, x\right) \neq\left(t_{2}, x\right) \in[0, T] \times \mathbb{T}^{d}} \frac{\left|v\left(t_{1}, x\right)-v\left(t_{2}, x\right)\right|}{\left|t_{1}-t_{2}\right|^{\frac{1+\beta}{2}}} .
$$


We set $C^{1,2}\left([0, T] \times \mathbb{T}^{d} ; \mathbb{R}\right)$ to be the set of functions which admit first derivative with resepct to time and second derivatives with respect to the state variables, such that these derivatives are continuous with respect to time and state.

Throughout the paper, what we call a solution to (1.2) is precisely defined by the following definition.

Definition 2.1. The triple $(u, m, \mu)$ is a solution to (1.2) if $u \in C^{1,2}\left([0, T] \times \mathbb{T}^{d}\right)$ is a pointwise solution to the Hamilton-Jacobi-Bellman equation (1.2a) with terminal condition (1.2d), $m \in$ $C^{0}\left([0, T] \times \mathbb{T}^{d} ; \mathbb{R}\right)$ is solution to the Fokker-Planck-Kolmogorov equation (1.2b) in the sense of distribution with initial condition (1.2e) , and $\mu \in C^{0}\left([0, T] ; \mathcal{P}_{\infty}\left(\mathbb{T}^{d} \times \mathbb{R}^{d}\right)\right)$ satisfies (1.2C) at any $t \in[0, T]$.

A simple way to overcome difficulty 2) is to assume that the Hamiltonian $H$ and some of its derivatives admit uniform bounds with respect to $\mu$. In this case, the well-posedness of the MFGC system with a possibly degenerate diffusion is investigated in [11]. Here we avoid such an assumption for (1.2) but we introduce the following approximating system which satisfies it,

$(2.4 \mathrm{a})-\partial_{t} u^{M}(t, x)-\nu \Delta u^{M}(t, x)+H\left(x, \nabla_{x} u^{M}(t, x), \mu^{M}(t)\right)=0$

(2.4b) $\partial_{t} m_{t}^{M}(t, x)-\nu \Delta m^{M}(t, x)-\operatorname{div}\left(H_{p}\left(x, \nabla_{x} u^{M}(t, x), \mu^{M}(t)\right) m^{M}\right)=0$

(2.4c) $\mu^{M}(t)=\left[I_{d}, T_{M}\left(-H_{p}\left(\cdot, \nabla_{x} u^{M}(t, \cdot), \mu^{M}(t)\right)\right)\right] \# m^{M}(t)$

$(2.4 \mathrm{~d}) u^{M}(T, x)=g\left(x, m^{M}(T)\right)$

(2.4e) $m^{M}(0)=m_{0}$, in $(0, T) \times \mathbb{T}^{d}$,

in $(0, T) \times \mathbb{T}^{d}$,

in $[0, T]$,

in $\mathbb{T}^{d}$,

where $M$ is a positive constant and $T_{M}$ is a truncation map defined by

$$
T_{M}(v)=\left\{\begin{array}{l}
v \text { if }|v| \leq M \\
\frac{M}{|v|} v \text { otherwise }
\end{array}\right.
$$

The latter definition can be naturally extended to the case when $M=\infty$ by taking $T_{\infty}=I d_{\mathbb{R}^{d}}$. In this case systems (1.2) and (2.4) coincide. A solution to (2.4) is defined by replacing (1.2) by (2.4) in Definition 2.1.

If $M<\infty$ and $\left(u^{M}, m^{M}, \mu^{M}\right)$ is a solution to (2.4), $\mu^{M}(t)$ is compactly supported in $B_{\mathbb{R}^{d}}(0, M)$ the closed ball in $\mathbb{R}^{d}$ centered at 0 with radius $M$, for any $t \in[0, T]$. Consequently, $u^{M}$ and $m^{M}$ should satisfy estimates depending on $M$ and uniform with respect to $\mu^{M}$. Therefore, compactness results for (2.4) should be less demanding than for (1.2) and difficulty 2) should vanish.

\subsection{Assumptions}

Let us start with some reasonable assumptions about the regularity and the boundedness of the Hamiltonian, the terminal cost and the inital distribution of agents. We introduce two constants: $C_{0}>0$ and $\beta_{0} \in(0,1)$.

A1 $H=H(x, p, \mu)$ is convex with respect to $p$, and differentiable with respect to $(x, p) ; H_{p}$ is locally $\beta_{0}$-Hölder continuous with respect to $p ; H$ and $H_{p}$ are continuous with respect to $\mu$ on $\mathcal{P}_{\infty, R}\left(\mathbb{T}^{d} \times \mathbb{R}^{d}\right)$ for any $R>0$, where $\mathcal{P}_{\infty, R}\left(\mathbb{T}^{d} \times \mathbb{R}^{d}\right)$ is defined in paragraph 2.1 and equipped with the weak* topology.

A2 $g: \mathbb{T}^{d} \times \mathcal{P}\left(\mathbb{T}^{d}\right) \rightarrow \mathbb{R}$ is continuous, and we suppose that $x \mapsto g(x, m)$ is in $C^{2+\beta_{0}}\left(\mathbb{T}^{d}\right)$, with a norm bounded uniformly with respect to $m$, i.e.

$$
\|g(\cdot, m)\|_{C^{2+\beta_{0}}} \leq C_{0}, \quad \forall m \in \mathcal{P}\left(\mathbb{T}^{d}\right) .
$$


A3 $m_{0} \in \mathcal{P}\left(\mathbb{T}^{d}\right)$ is absolutely continuous with respect to the Lebesgue measure on $\mathbb{T}^{d}$ and we also name $m_{0}$ its density (abuse of notation). Assume that $m_{0} \in C^{\beta_{0}}\left(\mathbb{T}^{d}\right)$ and is positive (see Remark 2.2 below to drop out the positivity assumption).

These assumptions are not restrictive when looking for solutions with the regularity given in Definition 2.1. However, they can be relaxed if we are interested in weaker solutions of systems (1.2).

In this paper we consider nonlocal coupling through the controls. More precisely, we assume that these interactions involve the quantity $\Lambda_{q_{0}}(\mu)$ defined in (2.1).

Let us introduce the assumptions used to address difficulty 1) which consists of solving the fixed point relations in $\mu$ given in (1.2c) and (2.4c), when $(u, m)$ are fixed and have the same regularity as in Definition 2.1. We introduce $\lambda_{0} \in[0,1)$, for all $(x, p, m) \in \mathbb{T}^{d} \times \mathbb{R}^{d} \times \mathcal{P}\left(\mathbb{T}^{d}\right)$, and $\mu, \mu^{1}, \mu^{2} \in \mathcal{P}_{m}\left(\mathbb{T}^{d} \times \mathbb{R}^{d}\right)$, we assume that,

FP1 $\left|H_{p}(x, p, \mu)\right| \leq C_{0}\left(1+|p|^{q-1}\right)+\lambda_{0} \Lambda_{q_{0}}(\mu)$.

FP2 $\left|H_{p}\left(x, p, \mu^{1}\right)-H_{p}\left(x, p, \mu^{2}\right)\right| \leq \lambda_{0}\left\|\alpha^{\mu^{1}}-\alpha^{\mu^{2}}\right\|_{L^{q_{0}(m)}}$.

These structural assumptions for MFGC are new in the literature and participate to the originality and novelty of the results presented in this paper. Moreover they do not seem to be restrictive as it is explained in what follows.

We recall that the optimal control of a representative agent is given by $\alpha=-H_{p}\left(x, \nabla_{x} u, \mu\right)$. Since $\Lambda_{q_{0}}(\mu)$ is homogeneous to the norm of a control, we cannot expect the dependency of $H_{p}$ upon $\mu$ to involve an exponent larger than one. Moreover if $m$ is the first marginal of $\mu$, taking the $L^{q_{0}}(m)$-norm in FP1 makes $\Lambda_{q_{0}}(\mu)$ appear in both sides of the resulting inequality; this explains the form of the right-hand side in FP1 and the necessity of choosing $\lambda_{0}$ smaller than 1. Similar arguments can provide insights on FP2, by noticing that if $\Lambda_{q_{0}}$ was seen as a norm on $\mathcal{P}_{m}\left(\mathbb{T}^{d} \times \mathbb{R}^{d}\right)$ then $\left\|\alpha^{\mu^{1}}-\alpha^{\mu^{2}}\right\|_{L^{q_{0}(m)}}$ would be the associated distance. We refer to Remark 4.3 in [1] for a concrete example of a MFGC system which does not admit solution if $\lambda_{0}=1$.

As in a large part of the literature on MFG or HJB equations, we consider Hamiltonians that are power-like functions in $p$ at least asymptotically. Let $q \in(1, \infty)$ be this asymptotic exponent, and $q^{\prime}$ the conjugate exponent of $q$ defined by $q^{\prime}=\frac{q}{q-1}$. Namely, we assume that $H$ satisfies the following inequalities, for all $x \in \mathbb{T}^{d}, p \in \mathbb{R}^{d}, m \in \mathcal{P}\left(\mathbb{T}^{d}\right)$, and $\mu \in \mathcal{P}_{m}\left(\mathbb{T}^{d} \times \mathbb{R}^{d}\right)$,

B1 $|H(x, 0, \mu)| \leq C_{0}+\lambda_{2} \Lambda_{q_{0}}(\mu)^{q^{\prime}}$, with $\lambda_{2} \geq 0$.

B2 $\left|H_{x}(x, p, \mu)\right| \leq C_{0}\left(1+|p|^{q}+\Lambda_{q_{0}}(\mu)^{q^{\prime}}\right)$.

B3 $H_{p}(x, p, \mu) \cdot p-H(x, p, \mu) \geq C_{0}^{-1}\left(|p|^{q}-\lambda_{1} \Lambda_{q_{0}}(\mu)^{q^{\prime}}\right)-C_{0}$, where $\lambda_{1}$ is a nonnegative constant satisfying $0 \leq \lambda_{1}<\frac{\left(1-\lambda_{0}\right)^{q^{\prime}}}{C_{0}^{q^{\prime}}}$.

One may notice that the dependencies of $H$ upon $p$ and $\mu$ involve different exponents (which happen to be equal when $q=2$ ). Indeed the Legendre transform applied to a power-like function make the exponent change into its conjugate. Since $H$ is defined as the Legendre transform of the Lagrangian $L$, the exponent in the dependency of $L$ upon $\alpha$ should be $q^{\prime}$. Moreover, $\Lambda_{q_{0}}(\mu)$ is homogeneous to the norm of a control, therefore $L$ should at most involve $\Lambda_{q_{0}}(\mu)^{q^{\prime}}$. Going back to the Hamiltonian by the Legendre transform, the exponent on $\Lambda_{q_{0}}(\mu)$ stays the same which explains the right-hand side in B1 B3. One may find the abovementionned growth conditions on $L$ in 28 .

Assumption B3 is a convexity property of $H$ with respect to $p$. In MFG without coupling through the controls, such an assumption is common, the only difference is that the term in 
$\Lambda_{q_{0}}(\mu)$ does not appear. This assumption will be particularly useful to obtain energy integral estimates by taking advantage of the duality properties of the forward-backward systems (1.2a), $(1.2 \mathrm{~b})$ and (2.4a), (2.4b). The inequality satisfied by $\lambda_{1}$ is needed in the calculation for getting these estimates. Let us mention that the right-hand side in this inequality comes from the estimates in Lemma 3.1. and that the constant $C_{0}$ can be identified with the one in FP1.

In order to obtain classical solutions of the HJB equations (1.2a) and (2.4a), we need Hölder continuity of $(t, x) \mapsto H\left(x, \nabla_{x} u(t, x), \mu(t)\right)$. While the space regularity of the latter map is straightforward here, its time regularity may be more demanding and we need assumptions which allow one to compare $H$ at different measures $\mu^{1}, \mu^{2} \in \mathcal{P}_{\infty}\left(\mathbb{T}^{d} \times \mathbb{R}^{d}\right)$. Assumption FP2 is not enough since it requires $\mu^{1}$ and $\mu^{2}$ to share the same marginal with respect to $\mathbb{T}^{d}$.

T For $R>0$, there exists a constant $C_{R}>0$ such that

$$
\begin{aligned}
\left|H\left(x, p, \mu^{1}\right)-H\left(x, p, \mu^{2}\right)\right| & \leq C_{R}\left(\left\|m^{1}-m^{2}\right\|_{\infty}^{\beta_{0}}+\left\|\alpha^{\mu^{1}}-\alpha^{\mu^{2}}\right\|_{\infty}\right), \\
\left|H_{p}\left(x, p, \mu^{1}\right)-H_{p}\left(x, p, \mu^{2}\right)\right| & \leq C_{R}\left(\left\|m^{1}-m^{2}\right\|_{\infty}^{\beta_{0}}+\left\|\alpha^{\mu^{1}}-\alpha^{\mu^{2}}\right\|_{\infty}\right),
\end{aligned}
$$

for $\left(x, p, m^{i}, \mu^{i}\right)$ such that $(x, p) \in \mathbb{T}^{d} \times \mathbb{R}^{d}$ with $|p| \leq R, m^{i} \in \mathcal{P}\left(\mathbb{T}^{d}\right) \cap C^{0}\left(\mathbb{T}^{d}\right)$ with $m^{i} \geq R^{-1}, \mu^{i} \in \mathcal{P}_{m^{i}}\left(\mathbb{T}^{d} \times \mathbb{R}^{d}\right)$ with $\alpha^{\mu^{i}} \in C^{0}\left(\mathbb{T}^{d} \times \mathbb{R}^{d}\right)$ and $\left\|\alpha^{\mu^{i}}\right\|_{\infty} \leq R, i=1,2$.

One may notice that when $\mu^{1}$ and $\mu^{2}$ have the same first marginal with respect to $\mathbb{T}^{d}$ the second inequality in T is implied by FP2. If one is only interested in weak solution to (1.2), $\mathbf{T}$ can be removed.

Remark 2.2. Letting $C_{R}$ depends on $\left\|\left(m^{i}\right)^{-1}\right\|_{\infty}$ was motivated by models of population dynamics which are discussed in paragraphs 6.3 and 6.4. The drawback of this assumption is that we have to assume that the initial distribution of agents $m_{0}$ is positive.

All the results in this paper hold if we do not assume $m_{0}$ to be positive in $\mathbf{A 3}$, and we remove the condition $m^{i} \geq R^{-1}$ in $\mathbf{T}$.

\subsection{Main results}

We recall that assumptions FP1 and FP2 are designed to address difficulty 1), and T to obtain time regularity of the fixed point $\mu$ in $1.2 \mathrm{c}$ or $2.4 \mathrm{C}$. More precisely, we state the following lemma that will be proved in Section 3 .

Lemma 2.3. Assume A1, FP1, FP2 and T. Take $p \in C^{\frac{\beta}{2}, \beta}\left([0, T] \times \mathbb{T}^{d} ; \mathbb{R}^{d}\right)$ and $m \in C^{\frac{\beta}{2}, \beta}\left([0, T] \times \mathbb{T}^{d}\right)$ such that $m \geq R^{-1}$ and $m(t) \in \mathcal{P}\left(\mathbb{T}^{d}\right)$ for $t \in[0, T]$, where $\beta \in(0,1)$ and $R>0$ are constants. For any $t \in[0, T]$, there exists a unique $\mu(t) \in \mathcal{P}\left(\mathbb{T}^{d} \times \mathbb{R}^{d}\right)$ satisfying

$$
\mu^{M}(t)=\left[I_{d}, T_{M}\left(-H_{p}\left(\cdot, p(t, \cdot), \mu^{M}(t)\right)\right)\right] \# m(t),
$$

where $M \in(0, \infty]$. Moreover, the $\operatorname{map}(t, x) \mapsto \alpha^{\mu^{M}(t)}(x)$ is in $C^{\frac{\beta \beta_{0}}{2}, \beta \beta_{0}}\left([0, T] \times \mathbb{T}^{d} ; \mathbb{R}^{d}\right)$, and its associated norm can be estimated from above by a constant which depends on $\|p\|_{C^{\frac{\beta}{2}, \beta}},\|m\|_{C^{\frac{\beta}{2}, \beta}}$, and the constants in the assumptions.

In Section 4, we prove the a priori estimates stated in the following lemma.

Lemma 2.4. Assume A1 A3, B2, B3, FP1, FP2 and T. If $(u, m, \mu)$ is a solution to (2.4) for $M \in(0, \infty]$, then

- $\left\|\nabla_{x} u\right\|_{\infty} \leq C\left(1+\|u\|_{\infty}\right)$ and $\|u\|_{\infty} \leq C\left(1+\left\|\nabla_{x} u\right\|_{\infty}^{q}\right)$, where $C$ is independent of $M$ and depends only on the constants in the assumptions, 
- $m$ is positive,

- $u \in C^{1+\frac{\beta}{2}, 2+\beta}\left([0, T] \times \mathbb{T}^{d}\right)$,

- $m \in C^{\frac{\beta}{2}, \beta}\left([0, T] \times \mathbb{T}^{d} ; \mathbb{R}\right)$,

- $(t, x) \mapsto \alpha^{\mu(t)}(x)$ is in $C^{\frac{\beta}{2}, \beta}\left([0, T] \times \mathbb{T}^{d} ; \mathbb{R}^{d}\right)$,

where $\beta \in\left(0, \beta_{0}^{2}\right)$. Moreover, $\left\|m^{-1}\right\|_{\infty}$ and the norms associated with the last three items above depend only on $\|u\|_{\infty},\left\|m_{0}^{-1}\right\|_{\infty}, \beta$ and the constants in the assumptions.

These estimates are weaker than their equivalents for MFG systems without interaction through controls. In particular, $u$ is not uniformly bounded in $\|\cdot\|_{\infty}$-norm. However, we believe that our estimate of $\left\|\nabla_{x} u\right\|_{\infty}$ is the best that we can achieve in our framework since its righthand side should be at least linear with respect to $\|u\|_{\infty}$. To our knowledge, such an estimate for systems of MFG with nonlocal dependency on $\nabla_{x} u$ (or more generally for MFG systems in which we do not have a uniform a priori estimate on $u$ ) is new in the literature.

Here, these a priori estimates are not sufficient to address the difficulty 2) and to obtain existence of solutions. However, existence can be obtained under several different kinds of assumptions; below, we supply a list of existence results under various assumptions:

Theorem 2.5. Assume A1,A3, B1,B3, FP1, FP2, T. There exists a solution to (1.2) if one of the following assertions is satisfied

a) $q_{0} \leq q^{\prime}$ and $|H(x, 0, \mu)| \leq C_{0}\left(1+\Lambda_{q_{0}}(\mu)^{\widetilde{q}}\right)$, where $\widetilde{q}$ is a constant satisfying $\widetilde{q}<q^{\prime}$ (Proposition 5.4),

b) $q_{0} \leq q^{\prime}$ and $\lambda_{1}+C_{0} \lambda_{2}<\frac{\left(1-\lambda_{0}\right)^{q^{\prime}}}{C_{0}^{q^{\prime}}}$ where $\lambda_{1}$ and $\lambda_{2}$ are respectively defined in $\mathbf{B} 3$ and $\mathbf{B} 1$, the $C_{0}$ on the left-hand side comes from $C_{0}^{-1}$ in $\mathbf{B 3}$ and the $C_{0}$ on the right-hand side comes from FP1 (Proposition 5.3),

c) $|H(x, 0, \mu)| \leq C_{0}\left(1+\Lambda_{q_{0}}(\mu)^{q^{\prime}-1}\right)$, for any $(x, \mu) \in \mathbb{T}^{d} \times \mathcal{P}\left(\mathbb{T}^{d} \times \mathbb{R}^{d}\right)$ (Proposition 5.5),

d) $\left|H_{x}(x, p, \mu)\right| \leq C_{0}\left(1+|p|+\Lambda_{q_{0}}(\mu)^{q^{\prime}-1}\right)$, for any $(x, p, \mu) \in \mathbb{T}^{d} \times \mathbb{R}^{d} \times \mathcal{P}\left(\mathbb{T}^{d} \times \mathbb{R}^{d}\right)$ (Proposition 5.6),

e) $T \leq T_{0}$, where $T_{0}$ is a constant depending on the constants in the assumptions (Proposition [5.8).

An other additional assumption under which existence holds is the monotonicity condition addressed in [28].

We also give a uniqueness result under a short time horizon assumption.

Theorem 2.6 (Uniqueness with short time horizon). Assume A1, A2, A3, B1, B2, B3, FP1, FP2, and that the following three assumptions are satisfied,

- $H_{p}$ is locally Lipschitz continuous with respect to $p$,

- $g$ satisfies

$$
\left\|g\left(\cdot, m^{1}\right)-g\left(\cdot, m^{2}\right)\right\|_{C^{1+\beta}} \leq C_{0} W_{q_{1}}\left(m^{1}, m^{2}\right),
$$

for any $m^{1}, m^{2} \in \mathcal{P}\left(\mathbb{T}^{d}\right)$, where $q_{1} \in[1, \infty)$ and $W_{q_{1}}$ is the $q_{1}$-Wassertein distance on measures, 
- the two inequalities in $\mathbf{T}$ hold when we replace $\left\|m^{1}-m^{2}\right\|_{\infty}^{\beta_{0}}$ by $W_{q_{1}}\left(m^{1}, m^{2}\right)$.

There exists $T_{1}>0$ such that if $T<T_{1}$ then there is at most one solution to (1.2).

We believe that this uniqueness result can be easily extended to more general Hamiltonians, but that the short-time assumption is essential. Indeed numerical examples in which non-uniqueness occurs are presented in [1. In these examples, we consider groups of agents who start from some crowded areas at time $t=0$, and travel through the domain to arrive at some target areas. Imposing a short time assumption in such an example results in the agents not trying to reach the targets at all. Indeed in this case the kinetic cost makes it more expensive for them to cross the domain very quickly before the end of the game than to do nothing and just wait passively at their starting point. For this reason we were not interested in finding less restrictive assumptions in Theorem [2.6. This theorem should be only seen as an example of uniqueness result with a short time horizon assumption. In particular we wanted the proof in paragraph 5.4 to stay simple.

Remark 2.7. $\quad$ i) In this work, we only consider MFGC systems in the d-dimensional torus $\mathbb{T}^{d}$. However, we believe that our existence results (Theorem 2.5) hold under the same assumptions on the Euclidean space $\mathbb{R}^{d}$, and that the method introduced in [28] to pass from $\mathbb{T}^{d}$ to $\mathbb{R}^{d}$ can applied here.

ii) We did not include the case $q=1$ in this work (i.e. when the Hamiltonian is Lipschitz continuous in $p$ ). In this case, systems (1.2) and (2.4) coincide when $M$ is large enough, therefore there exists a solution to (1.2) under assumptions A1, A3, B1,B3, FP1, FP2 and $\mathbf{T}$, by the same arguments as in Lemma 5.1.

\section{3 - The Fixed POINT RELATION in $\mu$ AND the PROOF of LEMma 2.3}

We recall that (1.2) and (2.4) conincide when $M=\infty$. Here, we take $M \in(0, \infty]$.

The following lemma takes advantage of the structural assumptions FP1 and FP2 to solve the fixed point relations (1.2c) and (2.4c) which consists of difficulty 1) . It also states a priori estimates on $\mu$ which will be of great use in the next section to obtain a priori estimates on $u$ and its derivatives.

Lemma 3.1. Assume A1, FP1 and FP2. Take $p \in C^{0}\left(\mathbb{T}^{d} ; \mathbb{R}^{d}\right)$, and $m \in \mathcal{P}\left(\mathbb{T}^{d}\right)$. The following two assertions are satisfied.

(i) There exists a unique $\mu^{M} \in \mathcal{P}\left(\mathbb{T}^{d} \times \mathbb{R}^{d}\right)$ such that

$$
\mu^{M}=\left[I_{d}, T_{M}\left(-H_{p}\left(\cdot, p(\cdot), \mu^{M}\right)\right)\right] \# m .
$$

For any $\widetilde{q} \in[1, \infty]$, it satisfies

$$
\Lambda_{\widetilde{q}}\left(\mu^{M}\right) \leq \frac{C_{0}}{1-\lambda_{0}}\left(1+\left\||p|^{q-1}\right\|_{L^{\max \left(q_{0}, \widetilde{q}\right)}(m)}\right) .
$$

(ii) The map $(p, m) \mapsto \mu^{M}$ given by (3.1), is continuous from $C^{0}\left(\mathbb{T}^{d} ; \mathbb{R}^{d}\right) \times \mathcal{P}\left(\mathbb{T}^{d}\right)$ to $\mathcal{P}\left(\mathbb{T}^{d} \times \mathbb{R}^{d}\right)$. We recall that the spaces of measures are equipped with the weak-* topology.

Proof. (i) Let us define the following map,

$$
\begin{aligned}
C^{0}\left(\mathbb{T}^{d} ; \mathbb{R}^{d}\right) \rightarrow C^{0}\left(\mathbb{T}^{d} ; \mathbb{R}^{d}\right) \\
\Phi_{(p, m)}^{M}: \\
\alpha \mapsto\left\{\begin{array}{l}
\mathbb{T}^{d} \rightarrow \mathbb{R}^{d} \\
x \mapsto T_{M}\left(-H_{p}\left(x, p(x),\left(I_{d}, \alpha\right) \# m\right)\right) .
\end{array}\right.
\end{aligned}
$$


This map is well defined by (A1). It is $\lambda_{0}$-Lipschitz continuous by FP2 and the fact that $T_{M}$ is 1-Lipschitz continuous, we recall that $\lambda_{0}<1$. Therefore it admits a unique fixed point by the Banach fixed point theorem. If $\mu^{M} \in \mathcal{P}\left(\mathbb{T}^{d} \times \mathbb{R}^{d}\right)$ satisfies (3.1) then $\alpha^{\mu^{M}}$ is the only fixed point of $\Phi_{(p, m)}^{M}$. Conversely, if we denote by $\alpha$ the fixed point of $\Phi_{(p, m)}^{M}$, then $\mu^{M}$ defined by $\mu^{M}=\left(I_{d}, \alpha\right) \# m$ satisfies (3.1). This implies that (3.1) admits a unique fixed point that we name $\mu^{M}$ in what follows. From FP1, $\mu^{M}$ satisfies,

$$
\begin{aligned}
\Lambda_{\widetilde{q}}\left(\mu^{M}\right) & =\left\|\alpha^{\mu^{M}}\right\|_{L^{\widetilde{q}}(m)} \\
& \leq\left\|C_{0}\left(1+|p|^{q-1}\right)+\lambda_{0} \Lambda_{q_{0}}\left(\mu^{M}\right)\right\|_{L^{\widetilde{q}}(m)} \\
& \leq C_{0}\left(1+\left\||p|^{q-1}\right\|_{L^{\widetilde{q}}(m)}\right)+\lambda_{0} \Lambda_{\widetilde{q}}\left(\mu^{M}\right),
\end{aligned}
$$

for $\widetilde{q} \geq q_{0}$, where we obtained the last line by using the triangle inequality for the $L^{\widetilde{q}}$-norm, and (2.2). This implies (3.2) for any $\widetilde{q} \geq q_{0}$. Then we extend this result to $1 \leq \widetilde{q}<q_{0}$ by combining (2.2) and (3.2) applied to $q_{0}$.

(ii) Let $\left(p^{n}, m^{n}\right)_{n \in \mathbb{N}} \in\left(C^{0}\left(\mathbb{T}^{d} \times \mathbb{R}^{d}\right) ; \mathcal{P}\left(\mathbb{T}^{d}\right)\right)^{\mathbb{N}}$ be a convergent sequence to $(p, m)$ in $C^{0}\left(\mathbb{T}^{d} ; \mathbb{R}^{d}\right) \times$ $\mathcal{P}\left(\mathbb{T}^{d}\right)$. We define $\mu^{N}$ as before, and $\left(\mu^{N, n}\right)_{n \in \mathbb{N}}$ the fixed points satisfying

$$
\mu^{N, n}=\left[I_{d}, T_{M}\left(-H_{p}\left(\cdot, p^{n}(\cdot), \mu^{N, n}\right)\right)\right] \# m^{n}
$$

for $n \in \mathbb{N}$. The sequence $\left(p^{n}\right)_{n \in \mathbb{N}}$ is bounded in $C^{0}\left(\mathbb{T}^{d} ; \mathbb{R}^{d}\right)$, thus (3.2) with $\widetilde{q}=\infty$ yields that $\left(\mu^{N, n}\right)_{n \in \mathbb{N}}$ are uniformly compactly supported. The sequence $\left(\mu^{N, n}\right)$ is compact in $\mathcal{P}\left(\mathbb{T}^{d} \times \mathbb{R}^{d}\right)$ endowed with the weak-* topology. Let $\widetilde{\mu}$ be the limit of a subsequence $\left(\mu^{N, \varphi(n)}\right)_{n \in \mathbb{N}}$, for $\varphi: \mathbb{N} \rightarrow \mathbb{N}$ an increasing function. By continuity of $H_{p}$ and $T_{M}$, we can pass to the limit in (3.3) taken at $\varphi(n)$ when $n$ tends to infinity, this gives that $\widetilde{\mu}$ satisfies the same fixed point relation as $\mu$. By uniqueness of this fixed point, we deduce that $\widetilde{\mu}=\mu$. This implies that the entire sequence $\left(\mu^{N, n}\right)$ tends to $\mu$.

Therefore the map $(p, m) \mapsto \mu^{M}$ is continuous from $C^{0}\left(\mathbb{T}^{d} ; \mathbb{R}^{d}\right) \times \mathcal{P}\left(\mathbb{T}^{d}\right)$ to $\mathcal{P}\left(\mathbb{T}^{d} \times \mathbb{R}^{d}\right)$.

In particular if $q_{0} \leq q^{\prime},(2.2)$ and (3.2) yield

$$
\Lambda_{q_{0}}(\mu) \leq \Lambda_{q^{\prime}}(\mu) \leq \frac{C_{0}}{1-\lambda_{0}}\left(1+\|p\|_{L^{q}(m)}^{q-1}\right),
$$

and then we use the inequality $(a+b)^{q^{\prime}} \leq \frac{a^{q^{\prime}}}{\theta^{q^{\prime}-1}}+\frac{b^{q^{\prime}}}{(1-\theta)^{q^{\prime}-1}}$ which holds for $a, b>0$ and for any $\theta \in(0,1)$, to obtain

$$
\Lambda_{q_{0}}(\mu)^{q^{\prime}} \leq \frac{C_{0}^{q^{\prime}}}{\left(1-\lambda_{0}\right)^{q^{\prime}}}\left(\theta^{1-q^{\prime}}+(1-\theta)^{1-q^{\prime}}\|p\|_{L^{q}(m)}^{q}\right) .
$$

If $q \in[1, \infty]$ without restriction, we obtain

$$
\Lambda_{q_{0}}(\mu)^{q^{\prime}} \leq \frac{C_{0}^{q^{\prime}}}{\left(1-\lambda_{0}\right)^{q^{\prime}}}\left(\theta^{1-q^{\prime}}+(1-\theta)^{1-q^{\prime}}\|p\|_{\infty}^{q}\right) .
$$

These latter three inequalities will be of great use in Section 4 for getting a priori estimates.

Given $(u, m)$ as regular as in definition 2.1, we can use Lemma 3.1 to prove that the fixed point relations (1.2c) and (2.4c) are well-posed, and that if $(u, m, \mu)$ is a solution to (1.2) or (2.4) then $\mu$ is continuous with respect to time. However, we need a better regularity in time to get classical solution of the HJB equations (1.2a) and (2.4a). In Lemma 3.2, we use T to obtain an estimate of the distance between two fixed points of (3.1) associated with different $(u, m)$. We will be particularly interested in using this estimate on a solution to (2.4) at different times. 
Lemma 3.2. Assume A1, FP1, FP2 and T. Take $p^{1}, p^{2} \in C^{0}\left(\mathbb{T}^{d} ; \mathbb{R}^{d}\right)$, and $m^{1}, m^{2} \in \mathcal{P}\left(\mathbb{T}^{d}\right) \cap$ $C^{0}\left(\mathbb{T}^{d} ; \mathbb{R}\right)$ some positive probability measures. We define $\mu^{1}, \mu^{2} \in \mathcal{P}\left(\mathbb{T}^{d} \times \mathbb{R}^{d}\right)$ as the fixed point in (i) in Lemma 3.1 associated with $\left(p^{1}, m^{1}\right)$ and $\left(p^{2}, m^{2}\right)$, respectively. There exists a constant C such that

$$
\left\|\alpha^{\mu^{1}}-\alpha^{\mu^{2}}\right\|_{\infty} \leq C\left(\left\|p^{1}-p^{2}\right\|_{\infty}^{\beta_{0}}+\left\|m^{1}-m^{2}\right\|_{\infty}^{\beta_{0}}\right)
$$

where $C$ depends on $\left\|p^{i}\right\|_{\infty},\left\|\left(m^{i}\right)^{-1}\right\|_{\infty}$, for $i=1,2$, and the constants in the assumptions.

Proof. We define $\widetilde{\mu}$ by $\widetilde{\mu}=\left(I_{d}, \alpha^{\mu^{1}}\right) \# m^{2}$. The triangle inequality and the fact that $T_{M}$ is a contraction imply that for any $x \in \mathbb{T}^{d}$,

$$
\begin{aligned}
&\left|\alpha^{\mu^{1}}(x)-\alpha^{\mu^{2}}(x)\right| \leq\left|H_{p}\left(x, p^{1}(x), \mu^{1}\right)-H_{p}\left(x, p^{2}(x), \mu^{2}\right)\right| \\
& \leq\left|H_{p}\left(x, p^{1}(x), \mu^{1}\right)-H_{p}\left(x, p^{1}(x), \widetilde{\mu}\right)\right| \\
&+\left|H_{p}\left(x, p^{1}(x), \widetilde{\mu}\right)-H_{p}\left(x, p^{1}(x), \mu^{2}\right)\right| \\
& \quad+\left|H_{p}\left(x, p^{1}(x), \mu^{2}\right)-H_{p}\left(x, p^{2}(x), \mu^{2}\right)\right| .
\end{aligned}
$$

The measures $\mu^{1}$ and $\widetilde{\mu}$ are the image measures by the same function $\left(I_{d}, \alpha^{\mu^{1}}\right)$, of $m^{1}$ and $m^{2}$ respectively. From $\mathbf{T}$, we obtain

$$
\left|H_{p}\left(x, p^{1}(x), \mu^{1}\right)-H_{p}\left(x, p^{1}(x), \widetilde{\mu}\right)\right| \leq C_{R}\left\|m^{1}-m^{2}\right\|_{\infty}^{\beta_{0}}
$$

where $R=\max \left(\left\|p^{i}\right\|_{\infty},\left\|\left(m^{i}\right)^{-1}\right\|_{\infty}\right)$ and $C_{R}$ is the constant defined in T. We recall that $\Lambda_{\infty}\left(\mu^{i}\right)$ can be estimated from above by a quantity which only depends on $\left\|p^{i}\right\|_{\infty}$ and the constants in the assumptions, by (3.2).

Since $\widetilde{\mu}$ and $\mu^{2}$ have the same marginal with respect to $\mathbb{T}^{d}$, FP2 yields that,

$$
\left|H_{p}\left(x, p^{1}(x), \widetilde{\mu}\right)-H_{p}\left(x, p^{1}(x), \mu^{2}\right)\right| \leq \lambda_{0}\left\|\alpha^{\mu^{1}}-\alpha^{\mu^{2}}\right\|_{\infty} .
$$

Then $H_{p}$ is locally $\beta_{0}$-Hölder continuous by $\mathbf{A}$ so,

$$
\left|H_{p}\left(x, p^{1}(x), \mu^{2}\right)-H_{p}\left(x, p^{2}(x), \mu^{2}\right)\right| \leq C\left\|p^{1}-p^{2}\right\|_{\infty}^{\beta_{0}},
$$

for some constant $C$. Combining the latter four inequalities, we obtain,

$$
\left\|\alpha^{\mu^{1}}-\alpha^{\mu^{2}}\right\|_{\infty} \leq C_{1}\left(\left\|p^{1}-p^{2}\right\|_{\infty}^{\beta_{0}}+\left\|m^{1}-m^{2}\right\|_{\infty}^{\beta_{0}}\right)+\lambda_{0}\left\|\alpha^{\mu^{1}}-\alpha^{\mu^{2}}\right\|_{\infty},
$$

which implies (3.7) up to replacing $C$ with $\left(1-\lambda_{0}\right)^{-1} \max \left(C, C_{R}\right)$.

Lemma 2.3 is a straightfoward consequence of Lemmas 3.1 and 3.2 .

\section{4 - A priori estimates AND the PRoof of Lemma 2.4}

Here we take $M \in(0, \infty]$, and $(u, m, \mu)$ a solution to (2.4) defined in Definition 2.1. We will look for estimates independent of $M$ which allow us to address difficulty 2), These a priori estimates imply compactness results and play an essential role in the proofs of existence in Section 5 . 


\subsection{A priori estimates on $u$}

When we consider MFG without interactions through controls and with bounded coupling function and terminal cost, we can apply the maximum principle on parabolic differential equations to (4.3) below and get an a priori estimates of $\|u\|_{\infty}$ which only depends on the constants in the assumptions. However, for MFGC systems and more generally for HJB equations with non-local interactions in $\nabla_{x} u$, it is not possible to get such a strong a priori estimate directly from the maximum principle. Instead we get (4.1) and (4.2) which involve non-local quantities depending on $\nabla_{x} u$.

Lemma 4.1. Under assumptions A1, A2, B1, FP1, FP2, and $q_{0} \leq q^{\prime}$, for $\theta \in(0,1) u$ satisfies,

$$
\|u\|_{\infty} \leq C_{0}(1+T)+\frac{\lambda_{2} C_{0}^{q^{\prime}}}{\left(1-\lambda_{0}\right)^{q^{\prime}}}\left(\theta^{1-q^{\prime}} T+(1-\theta)^{1-q^{\prime}} \int_{0}^{T} \int_{\mathbb{T}^{d}}\left|\nabla_{x} u\right|^{q} d m(t, x) d t\right)
$$

where $\lambda_{2}$ is defined in B1. More generally, for any $q_{0} \in[1, \infty]$ u satisfies,

$$
\|u\|_{\infty} \leq C_{0}(1+T)+\frac{\lambda_{2} C_{0}^{q^{\prime}}}{\left(1-\lambda_{0}\right)^{q^{\prime}}}\left(\theta^{1-q^{\prime}} T+(1-\theta)^{1-q^{\prime}}\left\|\nabla_{x} u\right\|_{\infty}^{q}\right) .
$$

Proof. Here, we can rewrite (2.4a) in the following way,

$$
-\partial_{t} u(t, x)-\nu \Delta u(t, x)+\left[\int_{0}^{1} H_{p}\left(x, s \nabla_{x} u, \mu(t)\right) d s\right] \cdot \nabla_{x} u(t, x)=-H(x, 0, \mu(t)),
$$

for $(t, x) \in(0, T) \times \mathbb{T}^{d}$. The maximum principle for parabolic second-order equation applies to $u$ and $-u$,

$$
\|u\|_{\infty} \leq\|u(T, \cdot)\|_{\infty}+\int_{0}^{T}\|H(\cdot, 0, \mu(t))\|_{\infty} d t
$$

Moreover, $|H(x, 0, \mu(t))| \leq C_{0}+\lambda_{2} \Lambda_{q_{0}}(\mu(t))^{q^{\prime}}$ and $|u(T, x)| \leq C_{0}$ come from B1 and A2. respectively. We combine the latter inequalities with (3.5) and (3.6) to get (4.1) when $q_{0} \leq q^{\prime}$, and (4.2) respectively.

The non-local term in (4.1) involving $\nabla_{x} u$ corresponds roughly speaking to an energy. Moreover this is a quantity that naturally appears in MFG literature thanks to duality properties in the forward-backward systems (1.1), (1.2), or (2.4). More precisely, the FPK equations is the dual equation of the linearized HJB equation with respect to $u$. Lemma 4.2 provides an a priori estimate of this quantity.

Lemma 4.2. Under assumptions A1, A2, B3, FP1, FP2, and $q_{0} \leq q^{\prime}$, the following inequality is satisfied,

$$
\begin{aligned}
\int_{0}^{T} \int_{\mathbb{T}^{d}}\left|\nabla_{x} u\right|^{q} d m(t, x) d t \leq \\
\quad\left(1-\frac{\lambda_{1} C_{0}^{q^{\prime}}}{(1-\theta)^{q^{\prime}}-1\left(1-\lambda_{0}\right)^{q^{\prime}}}\right)^{-1}\left(C_{0}\|u\|_{\infty}+C_{0}^{2}(1+T)+\frac{\lambda_{1} C_{0}^{q^{\prime}} T}{\theta^{q^{\prime}-1}\left(1-\lambda_{0}\right)^{q^{\prime}}}\right),
\end{aligned}
$$

for any $\theta \in(0,1)$ such that $\lambda_{1}<\frac{(1-\theta)^{q^{\prime}-1}\left(1-\lambda_{0}\right)^{q^{\prime}}}{C_{0}^{q^{\prime}}}$. 
Proof. We multiply (2.4a) by $-m$ and (2.4b) by $u$; we add up and integrate over $(0, T) \times \mathbb{T}^{d}$ the resulting quantities; after performing some integrations by part, we obtain

$$
\begin{aligned}
\int_{0}^{T} \int_{\mathbb{T}^{d}}\left[H_{p}\left(x, \nabla_{x} u(t, x), \mu(t)\right) \cdot \nabla_{x} u-H(x\right. & \left.\left., \nabla_{x} u(t, x), \mu(t)\right)\right] d m(t, x) d t \\
& =\int_{\mathbb{T}^{d}} u(0, x) d m^{0}(x)-\int_{\mathbb{T}^{d}} g(x, m(T)) d m(T, x),
\end{aligned}
$$

that we can combine with $\mathbf{B 3}$ and $\mathbf{A 2}$ to get,

$$
C_{0}^{-1} \int_{0}^{T} \int_{\mathbb{T}^{d}}\left|\nabla_{x} u\right|^{q} d m(t, x) d t \leq\|u\|_{\infty}+C_{0}(1+T)+C_{0}^{-1} \lambda_{1} \int_{0}^{T} \Lambda_{q_{0}}(\mu(t))^{q^{\prime}} d t .
$$

We integrate (3.5) over $(0, T)$,

$$
\int_{0}^{T} \Lambda_{q_{0}}(\mu(t))^{q^{\prime}} d t \leq \frac{C_{0}^{q^{\prime}}}{\left(1-\lambda_{0}\right)^{q^{\prime}}}\left(\theta^{1-q^{\prime}} T+(1-\theta)^{1-q^{\prime}} \int_{0}^{T} \int_{\mathbb{T}^{d}}\left|\nabla_{x} u\right|^{q} d m(t, x) d t\right),
$$

where we can choose $\theta \in(0,1)$ such that $\lambda_{1}<\frac{(1-\theta)^{q^{\prime}-1}\left(1-\lambda_{0}\right)^{q^{\prime}}}{C_{0}^{q^{\prime}}}$, since $\lambda_{1}$ satisfies the inequality in B3. The latter three inequalities imply (4.5).

Roughly speaking, Lemma 4.1 with $q_{0} \leq q^{\prime}$ and Lemma 4.2 provide opposite inequalities which may become complementary under a smallness condition on the parameters, implying a uniform estimate on $\|u\|_{\infty}$. This condition is explicitely given in the following corollary.

Corollary 4.3. Under Assumptions [A1, A2, B1, B3, FP1, FP2, $q_{0} \leq q^{\prime}$, and $\lambda_{1}+C_{0} \lambda_{2}<$ $\frac{\left(1-\lambda_{0}\right)^{q^{\prime}}}{C_{0}^{q^{\prime}}}, u$ is bounded by a quantity which only depends on the constants in the assumptions.

Proof. Combing (4.1) and (4.5) results in,

$$
\begin{aligned}
\|u\|_{\infty} & \leq \frac{\lambda_{2} C_{0}^{q^{\prime}}}{(1-\theta)^{q^{\prime}-1}\left(1-\lambda_{0}\right)^{q^{\prime}}} C_{0}\left(1-\frac{\lambda_{1} C_{0}^{q^{\prime}}}{(1-\theta)^{q^{\prime}-1}\left(1-\lambda_{0}\right)^{q^{\prime}}}\right)^{-1}\|u\|_{\infty}+C_{\theta} \\
& \leq C_{0} \lambda_{2}\left(\frac{(1-\theta)^{q^{\prime}-1}\left(1-\lambda_{0}\right)^{q^{\prime}}}{C_{0}^{q^{\prime}}}-\lambda_{1}\right)^{-1}\|u\|_{\infty}+C_{\theta}
\end{aligned}
$$

where $\theta \in(0,1)$ may be chosen such that $\lambda_{1}+C_{0} \lambda_{2}<\frac{(1-\theta)^{q^{\prime}-1}\left(1-\lambda_{0}\right)^{q^{\prime}}}{C_{0}^{q^{\prime}}}$, and $C_{\theta}$ is a positive constant depending on the constants in the assumptions and $\theta$. This implies

$$
\|u\|_{\infty} \leq\left(1-C_{0} \lambda_{2}\left(\frac{(1-\theta)^{q^{\prime}-1}\left(1-\lambda_{0}\right)^{q^{\prime}}}{C_{0}^{q^{\prime}}}-\lambda_{1}\right)^{-1}\right) C_{\theta},
$$

where $C_{0} \lambda_{2}\left(\frac{(1-\theta)^{q^{\prime}-1}\left(1-\lambda_{0}\right)^{q^{\prime}}}{C_{0}^{q^{\prime}}}-\lambda_{1}\right)^{-1}<1$, which concludes the proof.

Let us mention that in the assumption $\lambda_{1}+C_{0} \lambda_{2}<\frac{\left(1-\lambda_{0}\right)^{q^{\prime}}}{C_{0}^{q^{\prime}}}$ in Corollary 4.3, the constant $C_{0}$ in the left-hand side comes from the $C_{0}^{-1}$ in $\mathbf{B 3}$, and the $C_{0}$ in the right-hand side comes from FP1. 


\subsection{A priori estimates on $m$}

In order for the HJB equations (1.2a) and (2.4a) to admit classical solutions, we want $\mu$ to be regular in time. Since $m$ is the marginal of $\mu$ with respect to $\mathbb{T}^{d}$, we first prove that $m$ is regular in the following lemma. Moreover, we also prove that $m$ stays positive, which is required in Lemma 2.3 to obtain time regularity on $\mu$.

Lemma 4.4. Under assumptions A1, A3, FP1, FP2, $m$ is in $C^{\frac{\beta}{2}, \beta}\left([0, T] \times \mathbb{T}^{d} ; \mathbb{R}\right)$ for $\beta \in$ $\left(0, \beta_{0}\right)$ and its $C^{\frac{\beta}{2}, \beta}\left([0, T] \times \mathbb{T}^{d}, \mathbb{R}\right)$-norm can be estimated from above by a constant which depends on $\left\|m_{0}\right\|_{C^{\beta_{0}}},\left\|\nabla_{x} u\right\|_{\infty}, \beta$ and the constants in the assumptions.

Furthermore, $m$ is positive everywhere and admits a positive lower bound which only depends on $\left\|m_{0}^{-1}\right\|_{\infty},\left\|\nabla_{x} u\right\|_{\infty}$ and the constants in the assumptions.

Proof. The distribution of agents $m$ satisfies the second-order parabolic FPK equation (2.4b), which is supplemented with a $\beta_{0}$-Hölder continuous initial condition. Theorem 2.1 section $V .2$ in [30] states that $m$ is uniformly bounded by a constant which depends on $\left\|m_{0}\right\|_{\infty}$ and $\left\|H_{p}\left(\cdot, \nabla_{x} u, \mu\right)\right\|_{\infty}$. This, (FP1) and (3.4) yield that $m H_{p}\left(\cdot, \nabla_{x} u, \mu, m\right)$ is bounded by a constant which depends on $\left\|m_{0}\right\|_{\infty},\left\|\nabla_{x} u\right\|_{\infty}$ and the constant of the assumptions. Finally, Theorem 6.29 in 34 yields that $m \in C^{\frac{\beta}{2}, \beta}\left([0, T] \times \mathbb{T}^{d}\right)$ for $\beta \in\left(0, \beta_{0}\right)$, and its associated norm can be estimated from above by a constant which depends on $\left\|m_{0}\right\|_{C^{\beta_{0}}},\left\|\nabla_{x} u\right\|_{\infty}, \beta$ and the constants in the assumptions.

We define $T_{\varepsilon}=\inf \left(\left\{t \in[0, T],\left\|m(t)^{-1}\right\|_{\infty} \leq \varepsilon\right\} \cup\{T\}\right)$, for $0<\varepsilon<\left\|m_{0}^{-1}\right\|_{\infty}$. In particular $T_{\varepsilon}$ is positive, since we proved in the latter paragraph that $m$ is continuous. On $\left[0, T_{\varepsilon}\right] \times \mathbb{T}^{d}$ we define the function $n$ by $n=m^{-1}$, it satisfies the following partial differential equation in the sense of viscosity,

$$
\partial_{t} n-\nu \Delta n-\operatorname{div}(\alpha n)+2 \alpha \cdot \nabla_{x} n=-2 \nu \frac{\left|\nabla_{x} n\right|^{2}}{n}
$$

supplemented with the initial condition $n(0)=m_{0}^{-1}$, where $\alpha(t, x)=-H_{p}\left(x, \nabla_{x} u(t, x), \mu(t)\right)$ for $(t, x) \in[0, T] \times \mathbb{T}^{d}$. We define $\widetilde{n}$ as the unique weak solution of the following partial differential equation defined on $[0, T] \times \mathbb{T}^{d}$,

$$
\partial_{t} \tilde{n}-\nu \Delta \widetilde{n}-\operatorname{div}(\alpha \widetilde{n})+2 \alpha \cdot \nabla_{x} \widetilde{n}=0,
$$

supplemented with the initial condition $\widetilde{n}(0)=m_{0}^{-1}$. Theorem 2.1 section $V .2$ in [30] states that $\widetilde{n}$ is bounded from above by a constant which depends on $\left\|m_{0}^{-1}\right\|_{\infty},\|\alpha\|_{\infty}$ and $T$. Moreover, $n$ is a subsolution of the restriction of (4.6) to $\left[0, T_{\varepsilon}\right] \times \mathbb{T}^{d}$, with the same initial condition as $\widetilde{n}$. Therefore, by a comparison argument for second-order parabolic equations in divergence form (Theorem 9.7 in [34] for instance), $n$ and $\widetilde{n}$ satisfy $n \leq \widetilde{n}$. This implies that there exists $C$ a positive constant independent of $T_{\varepsilon}$, such that $\|n\|_{\infty} \leq C$. We conclude the proof by taking $\varepsilon=2^{-1} C^{-1}$ and recalling that $\|\alpha\|_{\infty}$ can be estimated from above using FP1 and (3.4).

\subsection{A priori estimates on derivatives of $u$}

Bernstein methods are useful tools when studying HJB equations or MFG systems. They allow one to obtain a priori estimates on $\nabla_{x} u$ by considering the partial differential equations satisfied by some well-chosen functions depending on $u$ and $\nabla_{x} u$. See for example the video of the lecture of P.L. Lions on November the 23rd 2018 [35], in which Bernstein estimates are derived for MFG systems without interactions through controls. More precisely, P.L. Lions used the function defined by $\left|\nabla_{x} u\right|^{2} e^{-\eta u}$, for small $\eta$. Here this method might work only if we knew a uniform estimates on $\|u\|_{\infty}$ and if $q=2$. After significant changes in the latter method, we can derive an estimate on $u$ which is weaker than the one for MFG without interactions through controls. Namely, we state that $\left\|\nabla_{x} u\right\|_{\infty}$ is bounded by a quantity that depends linearly on $\|u\|_{\infty}$ by studying the functions $w$ and $\varphi$ defined in (4.11) below. To our knowledge, such estimates for 
systems of MFG with nonlocal dependency on $\nabla_{x} u$ (or more generally for MFG systems in which we do not have a uniform a priori estimate on $u$ ) are new in the literature. We believe that this result may hold for more general HJB equations with nonlocal dependency on $\nabla_{x} u$.

Lemma 4.5. Under assumptions A1, A2, B2, B3, FP1 and $\mathrm{FP2}$, there exists $C>0$ depending only on the constants of the assumptions, such that

$$
\left\|\nabla_{x} u(t)\right\|_{\infty} \leq C\left(1+\max _{t \leq s \leq T}\|u\|_{\infty}\right),
$$

for any $t \in[0, T]$.

Proof. In what follows, we only prove that (4.7) holds for $t=0$, however the proof does not use additional information available at $t=0$ (the initial condition on $m$ for example), so it can be repeated for any $t \in[0, T]$ and the constant $C$ in (4.7) does not depend on $t$.

Here we wish to differentiate (2.4a) with respect to $x$; however we did not assume in Definition 2.1 enough regularity on $u$ for such an operation to have sense pointwisely on $(0, T) \times \mathbb{T}^{d}$. Especially the time derivative of $\nabla_{x} u$ and the third derivatives of $u$ with respect to $x$ are not required to exist. This leads us to introducing $\rho \in C^{\infty}\left(\left[-\frac{1}{2}, \frac{1}{2}\right)^{d}\right)$ a non-negative mollifier such that $\rho(x)=0$ if $|x| \geq \frac{1}{4}$ and $\int_{\mathbb{R}^{d}} \rho(x) d x=1$. We introduce $\rho^{\delta}=\delta^{-d} \rho(\dot{\bar{\delta}})$ and $u^{\delta}(t)=\rho^{\delta} \star u(t)$, for any $0<\delta<1$ and $t \in[0, T]$, where $\star$ denotes the convolution operator.

Thus $u^{\delta}$ depends smoothly on the state variable and its partial derivatives in space at any order have the same regularity in time as $u$, moreover it solves the following partial differential equation with final condition,

$$
\left\{\begin{array}{lr}
-\partial_{t} u^{\delta}(t, x)-\nu \Delta u^{\delta}(t, x)+\rho^{\delta} \star\left(H\left(\cdot, \nabla_{x} u(t, \cdot), \mu(t)\right)\right)(x)=0 & \text { in }(0, T) \times \mathbb{T}^{d}, \\
u^{\delta}(T, x)=\rho^{\delta} \star(g(\cdot, m(T, \cdot)))(x) & \text { in } \mathbb{T}^{d},
\end{array}\right.
$$

Let us take the gradient with respect to the state variable of the latter equation and the scalar product of the resulting equality with $\nabla_{x} u^{\delta}$,

$$
\begin{aligned}
-\frac{1}{2} \partial_{t}\left|\nabla_{x} u^{\delta}\right|^{2}-\nu \nabla_{x} u^{\delta} \cdot \Delta\left(\nabla_{x} u^{\delta}\right)+\nabla_{x} u^{\delta} & \cdot D_{x, x}^{2} u^{\delta} H_{p}\left(x, \nabla_{x} u^{\delta}, \mu\right) \\
& +\nabla_{x} u^{\delta} \cdot H_{x}^{\delta}\left(x, \nabla_{x} u, \mu\right)=\nabla_{x} u^{\delta} \cdot R^{\delta}(t, x),
\end{aligned}
$$

where $H^{\delta}$ and $R^{\delta}$ are defined by

$$
\begin{aligned}
& H^{\delta}(x, p, \mu)=\rho^{\delta} \star(H(\cdot, p(\cdot), \mu))(x), \\
& R^{\delta}(t, x)=D_{x, x}^{2} u^{\delta} H_{p}\left(x, \nabla_{x} u^{\delta}, \mu\right)-\rho^{\delta} \star\left(D_{x, x}^{2} u H_{p}\left(\cdot, \nabla_{x} u, \mu\right)\right) .
\end{aligned}
$$

By simple calculus, we notice that

$$
\begin{aligned}
\nabla_{x}\left|\nabla_{x} u^{\delta}\right|^{2} & =2 D_{x, x}^{2} u^{\delta} \nabla_{x} u^{\delta} \\
\Delta\left|\nabla_{x} u^{\delta}\right|^{2} & =2 \nabla_{x} u^{\delta} \cdot \Delta\left(\nabla_{x} u^{\delta}\right)+2\left|D_{x, x}^{2} u^{\delta}\right|^{2},
\end{aligned}
$$

that we can combine with (4.9) and obtain

$$
\begin{aligned}
-\frac{1}{2} \partial_{t}\left|\nabla_{x} u^{\delta}\right|^{2}-\frac{\nu}{2} \Delta\left|\nabla_{x} u^{\delta}\right|^{2}+\nu\left|D_{x, x}^{2} u^{\delta}\right|^{2} & +\frac{1}{2} \nabla_{x}\left|\nabla_{x} u^{\delta}\right|^{2} \cdot H_{p}\left(x, \nabla_{x} u^{\delta}, \mu\right) \\
& =-\nabla_{x} u^{\delta} \cdot H_{x}^{\delta}\left(x, \nabla_{x} u, \mu\right)+\nabla_{x} u^{\delta} \cdot R^{\delta}(t, x) .
\end{aligned}
$$

We define the functions $\varphi$ and $w^{\delta}$ by

$$
\begin{aligned}
& \varphi(v)=\exp \left(\exp \left(-\left(a+b\|u\|_{\infty}^{-1} v\right)\right)\right), \text { for }|v| \leq\|u\|_{\infty}, \\
& w^{\delta}(t, x)=\varphi\left(u^{\delta}(T-t, x)\right)\left|\nabla_{x} u^{\delta}\right|^{2}(T-t, x),
\end{aligned}
$$


where $a>1$ and $b>0$ are constants that will be defined below. The derivatives of $\varphi$ are given by

$$
\begin{aligned}
& \varphi^{\prime}(v)=-b\|u\|_{\infty}^{-1} e^{-\left(a+b\|u\|_{\infty}^{-1} v\right)} \varphi(v) \\
& \varphi^{\prime \prime}(v)=b^{2}\|u\|_{\infty}^{-2} e^{-\left(a+b\|u\|_{\infty}^{-1} v\right)}\left(1+e^{-\left(a+b\|u\|_{\infty}^{-1} v\right)}\right) \varphi(v)
\end{aligned}
$$

which implies that $\varphi$ and $\varphi^{\prime}$ satisfy,

$$
\begin{aligned}
& 1 \leq \varphi(v) \leq e^{e^{-a+b}} \\
& b\|u\|_{\infty}^{-1} e^{-a-b} \leq \frac{\left|\varphi^{\prime}(v)\right|}{\varphi(v)} \leq b\|u\|_{\infty}^{-1} e^{-a+b} .
\end{aligned}
$$

Roughly speaking, we introduced $a$ and $b$ in order to have $\|\varphi\|_{\infty}\left\|\varphi^{-1}\right\|_{\infty}$ and $\left\|\varphi^{\prime}\right\|_{\infty}\left\|\left(\varphi^{\prime}\right)^{-1}\right\|_{\infty}$ as close as possible to 1 . This will be achieved by taking $a$ large enough, and $b$ small enough.

For simplicity of the notations, we will omit to write the argument of $\varphi$ since it is always $u^{\delta}$.

The derivatives of $w^{\delta}$ verify the following equalities,

$$
\begin{aligned}
-\varphi \partial_{t}\left|\nabla_{x} u^{\delta}\right|^{2} & =\partial_{t} w^{\delta}+\frac{\varphi^{\prime}}{\varphi} w^{\delta} \partial_{t} u^{\delta}, \\
\varphi \nabla_{x}\left|\nabla_{x} u^{\delta}\right|^{2} & =\nabla_{x} w^{\delta}-\frac{\varphi^{\prime}}{\varphi} w^{\delta} \nabla_{x} u^{\delta}, \\
\varphi \Delta\left|\nabla_{x} u^{\delta}\right|^{2} & =\Delta w^{\delta}-\frac{\varphi^{\prime}}{\varphi} w^{\delta} \Delta u^{\delta}+2 \frac{\varphi^{\prime}}{\varphi} \nabla_{x} w^{\delta} \cdot \nabla_{x} u^{\delta}-\frac{\varphi^{\prime \prime} \varphi-2\left(\varphi^{\prime}\right)^{2}}{\varphi^{3}}\left(w^{\delta}\right)^{2} .
\end{aligned}
$$

We multiply (4.10) by $2 \varphi$ and use the latter equalities in the resulting relation,

$$
\begin{gathered}
\partial_{t} w^{\delta}-\nu \Delta w^{\delta}+\nabla_{x} w^{\delta} \cdot H_{p}\left(x, \nabla_{x} u^{\delta}, \mu\right)-2 \nu \frac{\varphi^{\prime}}{\varphi} \nabla_{x} w^{\delta} \cdot \nabla_{x} u^{\delta}+2 \nu \varphi\left|D_{x, x}^{2} u^{\delta}\right|^{2} \\
=\frac{\varphi^{\prime}}{\varphi} w^{\delta}\left[-\partial_{t} u^{\delta}-\nu \Delta u^{\delta}+\nabla_{x} u^{\delta} \cdot H_{p}\left(x, \nabla_{x} u^{\delta}, \mu\right)\right]-\nu \frac{\varphi^{\prime \prime} \varphi-2\left(\varphi^{\prime}\right)^{2}}{\varphi^{3}}\left(w^{\delta}\right)^{2} \\
-2 \varphi \nabla_{x} u^{\delta} \cdot H_{x}^{\delta}\left(x, \nabla_{x} u, \mu\right)+2 \varphi \nabla_{x} u^{\delta} \cdot R^{\delta}(t, x) .
\end{gathered}
$$

We can rewrite the first line of (4.8) in the following way,

$$
-\partial_{t} u^{\delta}-\nu \Delta u^{\delta}=-H\left(x, \nabla_{x} u^{\delta}, \mu\right)-Q^{\delta},
$$

where $Q^{\delta}$ is defined by,

$$
Q^{\delta}(t, x)=H^{\delta}\left(x, \nabla_{x} u(t), \mu(t)\right)-H\left(x, \nabla_{x} u^{\delta}(t, x), \mu(t)\right) .
$$

This and (4.14) imply that

$$
\begin{aligned}
\partial_{t} w^{\delta}-\nu \Delta w^{\delta}+\nabla_{x} w^{\delta} \cdot H_{p}\left(x, \nabla_{x} u^{\delta}, \mu\right)-2 \nu \frac{\varphi^{\prime}}{\varphi} \nabla_{x} w^{\delta} \cdot \nabla_{x} u^{\delta}+2 \nu \varphi\left|D_{x, x}^{2} u^{\delta}\right|^{2} \\
=\frac{\varphi^{\prime}}{\varphi} w^{\delta}\left[\nabla_{x} u^{\delta} \cdot H_{p}\left(x, \nabla_{x} u^{\delta}, \mu\right)-H\left(x, \nabla_{x} u^{\delta}, \mu\right)\right]-\nu \frac{\varphi^{\prime \prime} \varphi-2\left(\varphi^{\prime}\right)^{2}}{\varphi^{3}}\left(w^{\delta}\right)^{2} \\
\quad-2 \varphi \nabla_{x} u^{\delta} \cdot H_{x}^{\delta}\left(x, \nabla_{x} u, \mu\right)+2 \varphi \nabla_{x} u^{\delta} \cdot R^{\delta}(t, x)-\varphi^{\prime}\left|\nabla_{x} u^{\delta}\right|^{2} Q^{\delta}(t, x) .
\end{aligned}
$$

In the following we will estimate from above the right-hand side of the latter expression. We notice that the second term of the right-hand side is negative since

$$
\varphi^{\prime \prime} \varphi-2\left(\varphi^{\prime}\right)^{2} \geq 0 .
$$


We notice that $R^{\delta}$ and $Q^{\delta}$ are uniformly convergent to 0 as $\delta$ tends to 0 , so we can assume that,

$$
\left\|R^{\delta}\right\|_{\infty}+\left\|Q^{\delta}\right\|_{\infty} \leq \frac{\varepsilon}{2 e\left\|\nabla_{x} u\right\|_{\infty}+\left\|\varphi^{\prime}\right\|_{\infty}\left\|\nabla_{x} u\right\|_{\infty}^{2}},
$$

for $\delta$ small enough and depending on $\varepsilon>0$.

The first term in the last line of (4.15) can be bounded using $\mathbf{B 2}$,

$$
-2 \varphi \nabla_{x} u^{\delta} \cdot H_{x}^{\delta}\left(x, \nabla_{x} u, \mu\right) \leq 2 C_{0} \varphi\left\|\nabla_{x} u^{\delta}\right\|_{\infty}\left(1+\left\|\nabla_{x} u^{\delta}\right\|_{\infty}^{q}+\Lambda_{q_{0}}(\mu(t))^{q^{\prime}}\right) .
$$

In fact we are going to use the latter inequality to obtain (4.19) below, only by noticing that using (3.6), the right-hand side involves only terms with exponents in $\left\|w^{\delta}\right\|_{\infty}$ or $\left\|w^{0}\right\|_{\infty}$ not larger than $\frac{1+q}{2}$.

Then we use B3 on the first term of the right-hand side of (4.15) since $\varphi^{\prime}<0$,

$$
\frac{\varphi^{\prime}}{\varphi} w^{\delta}\left[\nabla_{x} u^{\delta} \cdot H_{p}\left(x, \nabla_{x} u^{\delta}, \mu\right)-H\left(x, \nabla_{x} u^{\delta}, \mu\right)\right] \leq-C_{0}^{-1} \frac{\varphi^{\prime}}{\varphi^{1+\frac{q}{2}}}\left(w^{\delta}\right)^{1+\frac{q}{2}}+C_{0} \frac{\left|\varphi^{\prime}\right|}{\varphi} w^{\delta}+C_{0}^{-1} \lambda_{1} \frac{\left|\varphi^{\prime}\right|}{\varphi} w^{\delta} \Lambda_{q_{0}}(\mu(t))^{q^{\prime}}
$$

The term involving $-\left(w^{\delta}\right)^{1+\frac{q}{2}}$ is a key element in this proof. On the one hand, it will allow us to cancel the term in $w^{\delta} \Lambda_{q_{0}}(\mu(t))^{q^{\prime}}$. On the other hand, we will use the fact that it has a larger exponent than any of the remaining terms.

From (3.6) and (4.13), we obtain

$$
\frac{\left|\varphi^{\prime}\right|}{\varphi} \Lambda_{q_{0}}(\mu(t))^{q^{\prime}} \leq b\|u\|_{\infty}^{-1} e^{-a+b} \frac{C_{0}^{q^{\prime}}}{\left(1-\lambda_{0}\right)^{q^{\prime}}}\left(\theta^{1-q^{\prime}}+(1-\theta)^{1-q^{\prime}}\left\|w^{0}\right\|_{\infty}^{\frac{q}{2}}\right),
$$

where $\theta \in(0,1)$ will be defined below. Then (4.13) implies,

$$
\frac{\left|\varphi^{\prime}\right|}{\varphi^{1+\frac{q}{2}}} \geq b\|u\|_{\infty}^{-1} e^{-a-b} e^{-\frac{q}{2} e^{-a+b}}
$$

Combining the latter six inequalities, (4.15), and the fact that $\left\|w^{\delta}\right\|_{\infty} \leq\left\|w^{0}\right\|_{\infty}$, we obtain the following partial differential inequality,

$$
\begin{aligned}
& \partial_{t} w^{\delta}-\nu \Delta w^{\delta}+\nabla_{x} w^{\delta} \cdot H_{p}\left(x, \nabla_{x} u^{\delta}, \mu\right)-2 \nu \frac{\varphi^{\prime}}{\varphi} \nabla_{x} w^{\delta} \cdot \nabla_{x} u^{\delta} \\
& \leq-C_{0}^{-1} b\|u\|_{\infty}^{-1} e^{-a-b} e^{-\frac{q}{2} e^{-a+b}}\left(w^{\delta}\right)^{1+\frac{q}{2}}+b\|u\|_{\infty}^{-1} e^{-a+b} \frac{\lambda_{1} C_{0}^{q^{\prime}-1}}{(1-\theta)^{1-q^{\prime}}\left(1-\lambda_{0}\right)^{q^{\prime}}}\left\|w^{0}\right\|_{\infty}^{1+\frac{q}{2}} \\
&+\varepsilon+C_{a, b, \theta}\left(1+\|u\|_{\infty}^{-1}\right)\left(1+\left\|w^{0}\right\|_{\infty}^{\frac{1+q}{2}}\right)
\end{aligned}
$$

where $C_{a, b, \theta}$ is a positive constant which only depends on the constants in the assumptions and in $(a, b, \theta)$. We systematically used the inequality $\left\|w^{0}\right\|_{\infty}^{r} \leq 1+\left\|w^{0}\right\|_{\infty}^{\frac{1+q}{2}}$ on every term of the form $\left\|w^{0}\right\|_{\infty}^{r}$ with $0<r<\frac{1+q}{2}$.

Let us mention the following result: the function $y^{+}$defined by $y^{+}=\max \left(y_{0}, K^{-\frac{1}{k}}\|f\|_{\infty}^{\frac{1}{k}}\right)$ is a super-solution of the following differential equation,

$$
\left\{\begin{array}{l}
y^{\prime}(t)=-K y(t)^{k}+f(t) \\
y(0)=y_{0}
\end{array}\right.
$$

posed on $[0, T]$, where $k$ and $y_{0}$ are positive constants and $f$ is a bounded positive function. 
This and $\|w(0)\|_{\infty} \leq e C_{0}^{2}$ which comes from $\mathbf{A 2}$ and (4.13), yield that a super-solution to (4.19) is given by

$$
\left[\frac{\lambda_{1} C_{0}^{q^{\prime}} e^{2 b} e^{\frac{q}{2} e^{-a+b}}}{(1-\theta)^{q^{\prime}-1}\left(1-\lambda_{0}\right)^{q^{\prime}}}\left\|w^{0}\right\|_{\infty}^{1+\frac{q}{2}}+C_{a, b, \theta}\left(1+\|u\|_{\infty}\right)\left(1+\left\|w^{0}\right\|_{\infty}^{\frac{1+q}{2}}+\varepsilon\right)\right]^{\frac{2}{2+q}},
$$

where we replace $C_{a, b, \theta}$ with $C_{a, b, \theta}+\left(e C_{0}^{2}\right)^{1+\frac{q}{2}}$.

From a comparison argument for parabolic second-order equation, $w^{\delta}$ is not larger than the latter expression. This result holds for $w^{0}$ by letting $\delta$ and $\varepsilon$ tend to 0 , thus $w^{0}$ verifies the following inequality,

$$
\left\|w^{0}\right\|_{\infty}^{1+\frac{q}{2}} \leq \frac{\lambda_{1} C_{0}^{q^{\prime}} e^{2 b} e^{\frac{q}{2} e^{-a+b}}}{(1-\theta)^{q^{\prime}-1}\left(1-\lambda_{0}\right)^{q^{\prime}}}\left\|w^{0}\right\|_{\infty}^{1+\frac{q}{2}}+C_{a, b, \theta}\left(1+\|u\|_{\infty}\right)\left(1+\left\|w^{0}\right\|_{\infty}^{\frac{1+q}{2}}\right) .
$$

By B3, we can choose $a>1$ large enough, $b>0$ and $\theta \in(0,1)$ small enough such that $\frac{\lambda_{1} C_{0}^{q^{\prime}} e^{2 b} e^{\frac{q}{2}} e^{-a+b}}{(1-\theta)^{q^{\prime}-1}\left(1-\lambda_{0}\right)^{q^{\prime}}}<1$. This implies

$$
\left\|w^{0}\right\|_{\infty}^{1+\frac{q}{2}} \leq C_{a, b, \theta}\left(1+\|u\|_{\infty}\right)\left(1+\left\|w^{0}\right\|_{\infty}^{\frac{1+q}{2}}\right)
$$

where we increased $C_{a, b, \theta}$ into $\left(1-\frac{\lambda_{1} C_{0}^{q^{\prime}+1} e^{2 b} e^{\frac{q}{2}} e^{-a+b}}{(1-\theta)^{q^{\prime}-1}\left(1-\lambda_{0}\right)^{q^{\prime}}}\right)^{-1} C_{a, b, \theta}$.

We make out two cases: the first case is when $\left\|w^{0}\right\|_{\infty}^{\frac{1}{2}} \leq 2 C_{a, b, \theta}\left(1+\|u\|_{\infty}\right)$. The second case is when $\left\|w^{0}\right\|_{\infty}^{\frac{1}{2}}>2 C_{a, b, \theta}\left(1+\|u\|_{\infty}\right)$. In the latter case, (4.20) implies that $\left\|w^{0}\right\|_{\infty}^{1+\frac{q}{2}} \leq$ $\frac{1}{2}\left\|w^{0}\right\|_{\infty}^{\frac{1}{2}}\left(1+\left\|w^{0}\right\|_{\infty}^{\frac{1+q}{2}}\right)$, which implies that $\left\|w^{0}\right\|_{\infty} \leq 1$. Therefore, in any of the two latter cases we obtain

$$
\left\|w^{0}\right\|_{\infty}^{\frac{1}{2}} \leq 1+2 C_{a, b, \theta}\left(1+\|u\|_{\infty}\right) .
$$

This and (4.13) yield (4.7) when $t=0$, this concludes the proof.

Now, we can combine the estimates obtained in this section with classical results on parabolic second-order equations and get further estimates of $u$ and its derivatives and on $m$.

Lemma 4.6. Assume A1, A2, B2, B3, FP1, FP2 and T. The function u is in $C^{1+\frac{\beta}{2}, 2+\beta}\left([0, T] \times \mathbb{T}^{d}\right)$ for any $\beta \in\left(0, \beta_{0}^{2}\right)$, where $\beta_{0}$ was introduced in the assumptions. Its $C^{1+\frac{\beta}{2}, 2+\beta}$-norm can be bounded by a quantity depending only on $\|u\|_{\infty}, \beta$, and the constants in the assumptions.

Proof. Lemma 4.5 states that $\left\|\nabla_{x} u\right\|_{\infty}$ is bounded by a quantity which depends on $\|u\|_{\infty}$ and the constants in the assumptions. So is $\Lambda_{q_{0}}(\mu)$ by (3.2). Then $u$ is the solution of the heat equation with a right-hand side equal to $-H\left(x, \nabla_{x} u, \mu\right)$ which is bounded in $L^{\infty}$. Classical results (see for example Theorem 6.48 in [34]) state that for any $\beta \in(0,1)$, the $C^{\frac{1}{2}+\frac{\beta}{2}, 1+\beta}$-norm of $u$ is bounded by a constant which depends on the $L^{\infty}$-norm of the right-hand side, the terminal condition, and $\beta$.

Lemma 4.4 yields that $m$ is in $C^{\frac{\beta}{2}, \beta}\left([0, T] \times \mathbb{T}^{d}\right)$ for $\beta \in\left(0, \beta_{0}\right)$, is positive, and that both its $C^{\frac{\beta}{2}, \beta}\left([0, T] \times \mathbb{T}^{d}\right)$-norm and its lower bound depend on $\|u\|_{\infty},\left\|m_{0}^{-1}\right\|_{\infty}, \beta$, and the constant of the assumptions.

Therefore, Lemma 2.3 yields that $\left[(t, x) \mapsto \alpha^{\mu(t)}(x)\right] \in C^{\frac{\beta \beta_{0}}{2}, \beta \beta_{0}}\left([0, T] \times \mathbb{T}^{d} ; \mathbb{R}^{d}\right)$.

From A1, $H$ is locally Lipschitz continuous with respect to $(x, p)$. This and T imply that $\left[(t, x) \mapsto H\left(t, \nabla_{x} u(t, x), \mu(t)\right)\right] \in C^{\frac{\beta \beta_{0}}{2}, \beta \beta_{0}}\left([0, T] \times \mathbb{T}^{d}\right)$. Thus $u$ is the solution of the backward 
heat equation with a right-hand side in $C^{\frac{\beta \beta_{0}}{2}, \beta \beta_{0}}$ supplemented with terminal condition in $C^{2+\beta_{0}}$. Classical results (see for instance Theorem 4.9 in [34]) yield that $u$ is in $C^{1+\frac{\beta \beta_{0}}{2}, 2+\beta \beta_{0}}$, and its $C^{1+\frac{\beta \beta_{0}}{2}, 2+\beta \beta_{0}}$-norm depends on $\|g(\cdot, m(T))\|_{C^{2+\beta_{0}}}$ and the $C^{\frac{\beta \beta_{0}}{2}, \beta \beta_{0}}$-norm of the right-hand side. We recall that $\beta$ is any constant in $\left(0, \beta_{0}\right)$. The proof of the lemma is complete.

Following precisely the dependencies in the above estimates, we obtain that the $C^{1+\frac{\beta \beta_{0}}{2}, 2+\beta \beta_{0}}$ norm of $u$ can be estimated from above by a constant which depends on $\|u\|_{\infty},\left\|m_{0}^{-1}\right\|_{\infty}, \beta$, and the constants in the assumptions.

The conclusions of Lemmas 4.1, 4.4, 4.5 and 4.6 are summarized in Lemma 2.4.

\section{5 - EXISTENCE AND UNIQUENESS RESUlTS UNDER ADDITIONAL ASSUMPTIONS}

\subsection{Solving the MFGC systems for $M<\infty$}

Lemma 5.1. Under assumptions A1, A3, B2, B3, FP1, FP2, T and $M \in(0, \infty)$, there exists at least one solution to (2.4).

Proof. For $(u, m) \in C^{0,1}\left([0, T] \times \mathbb{T}^{d} ; \mathbb{R}\right) \times C^{0}\left([0, T] ; \mathcal{P}\left(\mathbb{T}^{d}\right)\right)$, we define $\mu^{M} \in C^{0}\left([0, T] ; \mathcal{P}\left(\mathbb{T}^{d} \times \mathbb{R}^{d}\right)\right)$ by

$$
\mu^{M}(t)=\left[I_{d}, T_{M}\left(-H_{p}\left(\cdot, \nabla_{x} u(t, \cdot), \mu^{M}(t)\right)\right)\right] \# m(t) \text { in }[0, T],
$$

using Lemma 2.3. Then we define $u^{M}$ as the viscosity solution of the following backward HJB equation with a final condition,

$$
\left\{\begin{array}{l}
-\partial_{t} u^{M}(t, x)-\nu \Delta u^{M}(t, x)+H\left(x, \nabla_{x} u^{M}(t, x), \mu^{M}(t)\right)=0 \\
u^{M}(T, x)=g(x, m(t))
\end{array}\right.
$$

We can rewrite the first line of the latter system in the following way,

$$
-\partial_{t} u^{M}-\nu \Delta u^{M}+\nabla_{x} u^{M} \cdot \int_{0}^{1} H_{p}\left(x, s \nabla_{x} u^{M}, \mu^{M}(t)\right) d s=-H\left(x, 0, \mu^{M}(t)\right),
$$

where the right-hand side is bounded using $\Lambda_{\infty}(\mu(t)) \leq M$, $\mathbf{B 1}$ and (2.2). The maximum principle for second-order parabolic equation provides that $u^{M}$ is bounded. Here, the proof of Lemma 4.5 can be repeated to prove that $\left\|\nabla_{x} u\right\|_{\infty}$ is bounded by a constant which depends on $M$ and the constants in the assumptions. Then with the same argument as in Lemma 4.6, $u^{M}$ is bounded in $C^{\frac{1}{2}+\frac{\beta}{2}, 1+\beta}$-norm, for all $\beta \in(0,1)$.

We define $m^{M}$ as the solution in the sense of distributions of the following Fokker-PlanckKolmogorov equation with an initial condition,

$$
\left\{\begin{array}{l}
\partial_{t} m_{t}^{M}(t, x)-\nu \Delta m^{M}(t, x)+\operatorname{div}\left(b(t, x) m^{M}\right)=0 \quad \text { in }(0, T) \times \mathbb{T}^{d}, \\
m^{M}(0)=m_{0}
\end{array}\right.
$$

with $b(t, x)=-H_{p}\left(x, \nabla_{x} u^{M}(t, x), \mu^{M}(t)\right)$ which is a continuous function with respect to $(t, x)$. Using the same arguments as in Lemma 4.4, we get that $m \in C^{\frac{\beta}{2}, \beta}\left(\mathbb{T}^{d} ; \mathbb{R}\right)$ for $\beta \in\left(0, \beta_{0}\right)$.

Moreover, $\|u\|_{C^{\frac{1}{2}+\frac{\beta}{2}, 1+\beta}},\|m\|_{C^{\frac{\beta}{2}, \beta}}$ are bounded by a constant which depends on $M, \beta$ and the constants in the assumptions. The map $(u, m) \mapsto \mu^{M}$ is continuous from $C^{0,1}\left([0, T] \times \mathbb{T}^{d} ; \mathbb{R}\right) \times$ $C^{0}\left([0, T] ; \mathcal{P}\left(\mathbb{T}^{d}\right)\right)$ to $C^{0}\left([0, T] ; \mathcal{P}\left(\mathbb{T}^{d} \times \mathbb{R}^{d}\right)\right)$ by Lemma 3.1. The map $\left(m, \mu^{M}\right) \mapsto u^{M}$ is continuous from $C^{0}\left([0, T] ; \mathcal{P}\left(\mathbb{T}^{d}\right)\right) \times C^{0}\left([0, T] ; \mathcal{P}\left(\mathbb{T}^{d} \times \mathbb{R}^{d}\right)\right)$ to $C^{0,1}\left([0, T] \times \mathbb{T}^{d} ; \mathbb{R}\right)$ by the stability of the solutions of viscosity. The map $\left(u^{M}, \mu^{M}\right) \mapsto m^{M}$ is continuous from $C^{0,1}\left([0, T] \times \mathbb{T}^{d} ; \mathbb{R}\right) \times$ $C^{0}\left([0, T] ; \mathcal{P}\left(\mathbb{T}^{d} \times \mathbb{R}^{d}\right)\right)$ to $C^{0}\left([0, T] ; \mathcal{P}\left(\mathbb{T}^{d}\right)\right)$ by linearity of the FPK equation. 
Thus the map $(u, m) \mapsto\left(u^{M}, m^{M}\right)$ is continuous from $C^{0,1}\left([0, T] \times \mathbb{T}^{d} ; \mathbb{R}\right) \times C^{0}\left([0, T] ; \mathcal{P}\left(\mathbb{T}^{d}\right)\right)$ to itself. Its fixed points are exactly the solutions to (2.4). The image of this map is a subset of a convex compact set. Therefore, there exists a fixed point by Schauder theorem, see [19] Corollary 11.2.

Using the same arguments as in the proof of Lemma 4.6, such a fixed point $u$ satisfies $u \in C^{1+\frac{\beta}{2}, 2+\beta}\left([0, T] \times \mathbb{T}^{d} ; \mathbb{R}\right)$ for any $\beta \in\left(0, \beta_{0}^{2}\right)$.

Considering $M<\infty$ in (2.4) consists of enforcing the condition $\Lambda_{\infty}(\mu(t)) \leq M$, i.e. the fact that the support of $\mu(t)$ is embedded in the compact set $\mathbb{T}^{d} \times B_{\mathbb{R}^{d}}(0, M)$, for $t \in[0, T]$. Therefore, the interactions through controls are uniformly bounded. Lemma 5.1 relies on that to state the existence of solutions to (2.4). For $M=\infty$, we can not obtain such a uniform estimate by combining only the results of Section 4. However if such an estimate exists, the result of Lemma 5.1 holds for $M=\infty$ and yields the existence of solutions to (1.2). More precisely, if a solution to (2.4) satisfies $\Lambda_{\infty}(\mu(t))<M$ for any $t \in[0, T]$, then it is also a solution to (1.2). This is summarized in the following Corollary.

Corollary 5.2. Under the same assumptions as in Lemma [5.1, if, for any $M>0$, any solution $(u, m, \mu)$ to (2.4) satisfies $\|u\|_{\infty} \leq C$, or $\left\|\nabla_{x} u\right\|_{\infty} \leq C$, for some $C>0$, then there exists at least one solution to (1.2).

Proof. By Lemma 5.1, we define $(u, m, \mu)$ as a solution to (2.4) for $M \in(0, \infty)$ that will be defined later. By Lemma 2.4, assuming that $\|u\|_{\infty}$ is bounded is equivalent to assuming that $\left\|\nabla_{x} u\right\|_{\infty}$ is bounded. Therefore, without loss of generality, we can assume that $\left\|\nabla_{x} u\right\|_{\infty} \leq C$. From [FP1 and (3.4), we obtain

$$
\left\|H_{p}\left(x, \nabla_{x} u, \mu\right)\right\|_{\infty} \leq C_{0}\left(1+C^{q-1}\right)+\frac{\lambda_{0} C_{0}}{1-\lambda_{0}}\left(1+C^{q-1}\right) .
$$

We define $M=1+C_{0}\left(1+C^{q-1}\right)+\frac{\lambda_{0} C_{0}}{1-\lambda_{0}}\left(1+C^{q-1}\right)$, then the truncation $T_{M}$ leaves $-H_{p}\left(\cdot, \nabla_{x} u^{M}, \mu\right)$ unchanged. Hence $(u, m, \mu)$ is a solution to (1.2).

\subsection{Existence results when $q_{0} \leq q^{\prime}$}

When $q_{0} \leq q^{\prime}$, we can use integral energy estimates. More precisely, inequalities (4.1) and (4.5) hold. Therefore, the assumptions under which we can prove existence should be weaker than in the case $q_{0}>q^{\prime}$ in which we have less estimates at our disposal.

In particular, Corollary 4.3 provides a uniform estimate on $\|u\|_{\infty}$ under suitable assumptions. Corollary 5.2 then yields the existence of a solution to (1.2): hence we may state the following theorem:

Proposition $\mathbf{5 . 3}$ (Existence of solution with small non-linearities). Under assumptions A1 A3, B1, B3, FP1, FP2, T, $q_{0} \leq q^{\prime}$, and $\lambda_{1}+C_{0} \lambda_{2}<\frac{\left(1-\lambda_{0}\right)^{q^{\prime}}}{C_{0}^{q^{\prime}}}$, there exists at least one solution to (1.2).

Instead of assuming that the multiplicative parameters are small like in Proposition 5.3, we suppose in Propositions 5.5 below the exponent for the interactions through controls is in fact smaller than the one appearing in B1.

Proposition 5.4. Assume A1, A3, B2, B3, FP1, FP2, T, $q_{0} \leq q^{\prime}$, and that $H$ satisfies

$$
|H(x, 0, \mu)| \leq C_{0}\left(1+\Lambda_{q_{0}}(\mu)^{\widetilde{q}}\right),
$$

for $(x, \mu) \in \mathbb{T}^{d} \times \mathcal{P}\left(\mathbb{T}^{d} \times \mathbb{R}^{d}\right)$, where $\widetilde{q} \in\left[0, q^{\prime}\right)$ is a constant. There exists a solution to (1.2). 
Proof. Let $(u, m, \mu)$ be a solution to (2.4) for $M \in(0, \infty)$. From A2 (4.4) and the new assumption, we obtain that,

$$
\begin{aligned}
\|u\|_{\infty} & \leq C_{0}(1+T)+C_{0} \int_{0}^{T} \Lambda_{q_{0}}(\mu(t))^{\widetilde{q}} d t \\
& \leq C_{0}(1+T)+C_{0} T^{\frac{q^{\prime}-\widetilde{q}}{q^{\prime}}}\left(\int_{0}^{T} \Lambda_{q_{0}}(\mu(t))^{q^{\prime}} d t\right)^{\frac{\widetilde{q}}{q^{\prime}}},
\end{aligned}
$$

where the second line is obtained by a Hölder inequality, since $\widetilde{q}<q^{\prime}$. Let us recall that the inequality $(a+b)^{\frac{\tilde{q}}{q^{\prime}}} \leq a^{\frac{\widetilde{q}}{q^{7}}}+b^{\frac{\tilde{q}}{q^{\prime}}}$ holds for any $a, b>0$. The latter two inequalities and (3.5) with $\theta=\frac{1}{2}$ imply,

$$
\|u\|_{\infty} \leq C+C\left(\int_{0}^{T} \int_{\mathbb{T}^{d}}\left|\nabla_{x} u(t, x)\right|^{q} d m(t, x) d t\right)^{\frac{\widetilde{q}}{q^{\prime}}},
$$

where $C>0$ is a constant which depends on the constants in the assumptions. This and (4.5) yield that,

$$
\|u\|_{\infty} \leq C+C\|u\|_{\infty}^{\frac{\widetilde{q}}{q^{\prime}}}
$$

up changing the value of $C$. Let us make out two cases: the first case is when $\|u\|_{\infty} \leq(2 C)^{\frac{q^{\prime}}{q^{\prime}-\widetilde{q}}}$. The second case is when $\|u\|_{\infty}>(2 C)^{\frac{q^{\prime}}{q^{\prime}-\widetilde{q}}}$, which implies $\|u\|_{\infty} \leq C+\frac{1}{2}\|u\|_{\infty}$. In any of the two cases, $u$ is uniformly bounded with respect to $M$. The desired result then stems from Corollary 5.2 ,

\subsection{Existence results which do not need the assumption $q_{0}<q^{\prime}$}

Here, we do not make the assumption $q_{0} \leq q^{\prime}$. We can still obtain an existence result in the same spirit as the one provided in Proposition 5.4. In the following proposition, the exponent for the interactions through controls is assumed to be smaller than the one appearing in B1 or in Proposition [5.4.

Proposition 5.5. Assume A1, A3, B2, B3, FP1, FP2, T, and that $H$ satisfies

$$
|H(x, 0, \mu)| \leq C_{0}\left(1+\Lambda_{q_{0}}(\mu)^{q^{\prime}-1}\right)
$$

for any $(x, \mu) \in \mathbb{T}^{d} \times \mathcal{P}\left(\mathbb{T}^{d} \times \mathbb{R}^{d}\right)$. There exists a solution to (1.2).

Proof. Take $(u, m, \mu)$ a solution to (2.4) for $M \in(0, \infty)$. Let us combine (4.3), (3.6) for $\theta=\frac{1}{2}$, (4.7), (5.2), and the inequality $(a+b)^{\frac{1}{q}} \leq a^{\frac{1}{q}}+b^{\frac{1}{q}}$ which holds for $a, b>0$; this yields

$$
-\partial_{t} u-\nu \Delta u+\nabla_{x} u \cdot \int_{0}^{1} H_{p}\left(x, s \nabla_{x} u(t, x), \mu(t)\right) d s \leq C\left(1+\max _{t \leq s \leq T}\|u(s)\|_{\infty}\right),
$$

for a constant $C>0$ which depends only on the constants in the assumptions. We recall that $\|u(T)\|_{\infty} \leq C_{0}$ by $\mathbf{A 2}$. We consider $y_{+}, y_{-} \in C^{1}([0, T] ; \mathbb{R})$ defined as $y_{+}(t)=C t+C_{0} e^{C t}$ and $y_{-}(t)=-C t-C_{0} e^{C t}$ such that they are solution to the following differential equations

$$
\begin{aligned}
& \left\{\begin{array}{l}
y_{+}^{\prime}(t)=C\left(1+y_{+}(t)\right) \\
y_{+}(0)=C_{0},
\end{array}\right. \\
& \left\{\begin{array}{l}
y_{-}^{\prime}(t)=C\left(-1+y_{-}(t)\right) \\
y_{-}(0)=-C_{0} .
\end{array}\right.
\end{aligned}
$$


By a comparison argument for second-order parabolic equation we obtain,

$$
-C T-C_{0} e^{C T} \leq y_{-}(T-t) \leq u(t, x) \leq y_{+}(t) \leq C T+C_{0} e^{C T},
$$

for $(t, x) \in[0, T] \times \mathbb{T}^{d}$. Therefore $u$ is uniformly bounded with respect to $M$. The desired result then stems from Corollary 5.2 .

In Propotitions 5.4 and 5.5. we changed the exponent appearing in B1. In the following proposition, we assume a smaller exponent than the one appearing in B2 instead.

Proposition 5.6 (Existence with more restrictive assumptions on $H_{x}$ ). Assume A1 A3, B1, B3, FP1, FP2, T, and the following inequality,

$$
\left|H_{x}(x, p, \mu)\right| \leq C_{0}\left(1+|p|+\Lambda_{q_{0}}(\mu)^{q^{\prime}-1}\right)
$$

for any $(x, p, \mu) \in \mathbb{T}^{d} \times \mathbb{R}^{d} \times \mathcal{P}\left(\mathbb{T}^{d} \times \mathbb{R}^{d}\right)$. There exists at least one solution to (1.2).

Proof. Take $(u, m, \mu)$ a solution to (2.4), for $M \in(0, \infty)$.

First step: we prove the following inequality,

$$
\left\|\nabla_{x} u(t)\right\|_{\infty}^{q} \leq C\left(1+\sup _{t \leq s \leq T}\|u(s)\|_{\infty}\right),
$$

for any $t \in[0, T]$, where $C>0$ is a constant depending only on the constants in the assumptions. We will only prove this inequality for $t=0$, however the proof does not use the additional information available at $t=0$ (the initial condition on $m$ for example), so it can be repeated for any $t \in[0, T]$ and the constant $C$ in (5.5) does not depend on $t$.

We introduce $\varphi, w^{\delta}, a, b, \delta$ and $\varepsilon$ as in the proof of Lemma 4.5. Using (5.4) instead of B22, we obtain

$$
-2 \varphi \nabla_{x} u^{\delta} \cdot H_{x}^{\delta}\left(x, \nabla_{x} u, \mu\right) \leq 2 C_{0} \varphi\left\|\nabla_{x} u^{\delta}\right\|_{\infty}\left(1+\left\|\nabla_{x} u^{\delta}\right\|_{\infty}+\Lambda_{q_{0}}(\mu(t))^{q^{\prime}-1}\right) .
$$

From this and (3.2), one may notice that the right-hand side of the latter inequality only involves terms with exponents in $\left\|w^{\delta}\right\|_{\infty}$ or $\left\|w^{0}\right\|_{\infty}$ nor larger than $\frac{1}{2}\left(1+(q-1)\left(q^{\prime}-1\right)\right)=1$. This and the same arguments as in the proof of Lemma 4.5) between (4.18) and (4.19), lead to the following inequality,

$$
\begin{aligned}
& \partial_{t} w^{\delta}-\nu \Delta w^{\delta}+\nabla_{x} w^{\delta} \cdot H_{p}\left(x, \nabla_{x} u^{\delta}, \mu\right)-2 \nu \frac{\varphi^{\prime}}{\varphi} \nabla_{x} w^{\delta} \cdot \nabla_{x} u^{\delta} \\
& \leq-C_{0}^{-1} b\|u\|_{\infty}^{-1} e^{-a-b} e^{-\frac{q}{2} e^{-a+b}}\left(w^{\delta}\right)^{1+\frac{q}{2}}+b\|u\|_{\infty}^{-1} e^{-a+b} \frac{\lambda_{1} C_{0}^{q^{\prime}}}{(1-\theta)^{1-q^{\prime}}\left(1-\lambda_{0}\right)^{q^{\prime}}}\left\|w^{0}\right\|_{\infty}^{1+\frac{q}{2}} \\
&+\varepsilon+C_{a, b, \theta}\left(1+\|u\|_{\infty}^{-1}\right)\left(1+\left\|w^{0}\right\|_{\infty}\right),
\end{aligned}
$$

instead of (4.19), where the novelty is the exponent on $\left\|w^{0}\right\|_{\infty}$ at the last line which changed from $\frac{1+q}{2}$ to 1 . Then following the same steps as in the proof of Lemma 4.5 until the end, we obtain that,

$$
\left\|\nabla_{x} u\right\|_{\infty}^{q} \leq C_{a, b, \theta}\left(1+\|u\|_{\infty}\right) .
$$

This concludes the first step of the proof.

Second step: obtaining a uniform estimate on $u$. 
Using B1, (3.6) with $\theta=\frac{1}{2}$ and (5.5), we obtain that,

$$
\begin{aligned}
|H(x, 0, \mu(t))| & \leq C_{0}\left(1+\Lambda_{q_{0}}(\mu(t))^{q^{\prime}}\right) \\
& \leq 2 C_{0}+\frac{C_{0}^{q^{\prime}+1} 2^{q^{\prime}-1}}{(1-\lambda)^{q^{\prime}}}\left(1+\left\|\nabla_{x} u(t)\right\|_{\infty}^{q}\right) \\
& \leq C\left(1+\max _{t \leq s \leq T}\|u(s)\|_{\infty}\right),
\end{aligned}
$$

where the constant $C$ from the previous step may have been increased. This implies that $u$ satisfies the same partial differential inequality as in the proof of Proposition 5.5. namely (5.3). Therefore the same arguments as in Proposition 5.5 apply and we conclude that there exists a solution to (1.2).

Remark 5.7. Note that the exponent $q^{\prime}-1$ actually appears in several applications: for instance, the price impact model described in paragraph 6.2 in the quadratic case (i.e. $q=2$ ) with $\varepsilon=0$ (i.e. when the bidding and asking prices are equal), satisfies the assumptions in both Propositions 5.5 and 5.6] with an exponent exactly equal to $q^{\prime}-1$.

\subsection{Existence and uniqueness results with a short-time horizon assumption}

Under a short-time horizon assumption, existence and even uniqueness of solutions are wellknown in the MFG literature. Indeed, when the time horizon is small, one may obtain strong a priori estimates under non-restrictive assumptions. These estimates combined with Corollary 5.2 yield existence of solution to (1.2) as stated in the following proposition.

Proposition 5.8 (Existence with short time horizon). Assume A1, A2 B1, B2, FP1, FP2, and $\mathbf{T}$. There exists $T_{0}>0$ such that, if $T \leq T_{0}$ then there exists a solution to (1.2).

Proof. Take $(u, m, \mu)$ a solution to (2.4) for $M \in(0, \infty)$. We combine (4.3), FP1, (3.6), (4.7), and the convex inequality $(a+b)^{q} \leq 2^{q-1}\left(a^{q}+b^{q}\right)$, and we obtain

$$
-\partial_{t} u-\nu \Delta u+\nabla_{x} u \cdot \int_{0}^{1} H_{p}\left(x, s \nabla_{x} u(t, x), \mu(t)\right) d s \leq C\left(1+\max _{t \leq s \leq T}\|u(s)\|_{\infty}^{q}\right),
$$

where $C$ is a positive constant which depends only on the constants in the assumptions. We recall that $\|u(T)\|_{\infty} \leq C_{0}$ by $\mathbf{\mathbf { A 2 }}$, Let us consider the following differential equation,

$$
\left\{\begin{array}{l}
y^{\prime}(t)=C\left(1+y^{q}\right) \\
y(0)=C_{0} .
\end{array}\right.
$$

There exists $T_{0}>0$ such that the latter differential equation admits a bounded solution on $\left[0, T_{0}\right]$. We suppose that $T \leq T_{0}$, then $(t, x) \mapsto y(T-t)$ is a super-solution to (5.6). Hence by a comparison principle, we get that $u \leq y$. The same argument applies in order to prove that $u \geq-y$. Therefore $u$ is uniformly bounded with respect to $M$, and there exists a solution to (1.2) by Corollary 5.2.

We will now prove Theorem 2.6 which states that uniqueness is achieved under a short-time horizon assumption. We believe that this uniqueness result can be easily extended to more general Hamiltonians, but that the short-time assumption is essential. Indeed, numerical simulations in [1] show that uniqueness does not hold for the discrete MFGC system obtained by approximating (1.2) with finite differences; we believe that uniqueness does not hold for (1.2) either. Theorem 2.6. should be interpreted only as a simple example of uniqueness result with a short-time horizon assumption. 
Proof of Theorem 2.6. We suppose that $T_{1} \leq T_{0}$, where $T_{0}$ was defined in Proposition 5.8, so that a solution to (1.2) satisfies uniform estimates on $\|u\|_{\infty},\left\|u^{i}\right\|_{C^{1,2}}$ and $\left\|m^{i}\right\|_{C^{0}}$ by Lemma 4.6, for $i=1,2$. Take $\left(u^{1}, m^{1}, \mu^{1}\right)$ and $\left(u^{2}, m^{2}, \mu^{2}\right)$ two solutions to (1.2). We define $u=u^{1}-u^{2}$, $m=m^{1}-m^{2}$ and $\alpha=\alpha^{\mu^{1}}-\alpha^{\mu^{2}}$.

In this proof $C>0$ is a constant which may differ from line to line and depends only on the constants in the assumptions, $\left\|u^{i}\right\|_{C^{1,2}}$ and $\left\|m^{i}\right\|_{C^{0}}$, for $i=1,2$.

We can repeat the proof of Lemma 3.2 replacing $\left\|m^{1}-m^{2}\right\|_{\infty}^{\beta_{0}}$ and $\left\|p^{1}-p^{2}\right\|_{\infty}^{\beta_{0}}$ respectively with $W_{q_{1}}\left(m^{1}, m^{2}\right)$ and $\left\|p^{1}-p^{2}\right\|_{\infty}^{\beta_{0}}$ everywhere and we obtain that,

$$
\|\alpha(t)\|_{\infty} \leq C\left(\left\|\nabla_{x} u(t)\right\|_{\infty}+W_{q_{1}}\left(m^{1}(t), m^{2}(t)\right)\right),
$$

for any $t \in[0, T]$. Let us consider $X^{1}$ and $X^{2}$ two random processes defined by

$$
\begin{aligned}
d X_{t}^{1} & =\alpha^{\mu^{1}}\left(t, X^{1}\right) d t+\sqrt{2 \nu} d W_{t} \\
d X_{t}^{2} & =\alpha^{\mu^{2}}\left(t, X^{2}\right) d t+\sqrt{2 \nu} d W_{t} \\
X_{0}^{1} & =X_{0}^{2}=X^{0},
\end{aligned}
$$

where $X^{0}$ is a random variable on $\mathbb{T}^{d}$ with law $m^{0}$ and $W$ is a Brownian motion independent of $X^{0}$. The respective laws of $\left(X_{t}^{1}, \alpha^{\mu^{1}}\left(t, X_{t}^{1}\right)\right)$ and $\left(X_{t}^{2}, \alpha^{\mu^{2}}\left(t, X_{t}^{2}\right)\right)$ are $\mu^{1}(t)$ and $\mu^{2}(t)$. Then we obtain,

$$
\begin{aligned}
\mathbb{E}\left[\left|X_{t}^{1}-X_{t}^{2}\right|^{q_{1}}\right]^{\frac{1}{q_{1}}} & =\mathbb{E}\left[\left|\int_{0}^{t} \alpha^{\mu^{1}}\left(s, X_{s}^{1}\right)-\alpha^{\mu^{2}}\left(s, X_{s}^{2}\right) d s\right|^{q_{1}}\right]^{\frac{1}{q_{1}}} \\
& \leq \int_{0}^{t} \mathbb{E}\left[\left|\alpha^{\mu^{1}}\left(s, X_{s}^{1}\right)-\alpha^{\mu^{2}}\left(s, X_{s}^{2}\right)\right|^{q_{1}}\right]^{\frac{1}{q_{1}}} d s \\
& \leq \int_{0}^{t} \mathbb{E}\left[\left|\alpha^{\mu^{1}}\left(s, X_{s}^{1}\right)-\alpha^{\mu^{1}}\left(s, X_{s}^{2}\right)\right|^{q_{1}}\right]^{\frac{1}{q_{1}}}+\mathbb{E}\left[\left|\alpha^{\mu^{1}}\left(s, X_{s}^{2}\right)-\alpha^{\mu^{2}}\left(s, X_{s}^{2}\right)\right|^{q_{1}}\right]^{\frac{1}{q_{1}}} d s
\end{aligned}
$$

where we used the triangle inequality for the $L^{q_{1}}$-norm twice. By the first additional assumption of the theorem and A1 $\alpha^{\mu^{1}}$ is Lipschitz continuous with respect to $x$ and its Lipschitz constant depends on $\left\|u^{i}\right\|_{C^{0,1}}$ and $\Lambda_{\infty}\left(\mu^{1}\right)$. Using the estimates from the proof of Proposition 5.8, it only depends on the constants in the assumptions. This, the latter inequality and (5.7) imply

$$
\mathbb{E}\left[\left|X_{t}^{1}-X_{t}^{2}\right|^{q_{1}}\right]^{\frac{1}{q_{1}}} \leq C \int_{0}^{t} \mathbb{E}\left[\left|X_{s}^{1}-X_{s}^{2}\right|^{q_{1}}\right]^{\frac{1}{q_{1}}} d s+C T \sup _{0 \leq t^{\prime} \leq T}\left(\left\|\nabla_{x} u\left(t^{\prime}\right)\right\|_{\infty}+W_{q_{1}}\left(m^{1}\left(t^{\prime}\right), m^{2}\left(t^{\prime}\right)\right)\right) .
$$

This and Gronwall's inequality yield that,

$$
\sup _{0 \leq t \leq T} \mathbb{E}\left[\left|X_{t}^{1}-X_{t}^{2}\right|^{q_{1}}\right]^{\frac{1}{q_{1}}} \leq C T \sup _{0 \leq t \leq T}\left(\left\|\nabla_{x} u(t)\right\|_{\infty}+W_{q_{1}}\left(m^{1}(t), m^{2}(t)\right)\right) .
$$

From now on, we assume that $T \leq \frac{1}{2 C}$, so that $(1-C T) \geq \frac{1}{2}$. Since $W_{q_{1}}\left(m^{1}(t), m^{2}(t)\right) \leq$ $\mathbb{E}\left[\left|X_{t}^{1}-X_{t}^{2}\right|^{q_{1}}\right]^{\frac{1}{q_{1}}}$, we obtain:

$$
\sup _{0 \leq t \leq T} W_{q_{1}}\left(m^{1}(t), m^{2}(t)\right) \leq C T \sup _{0 \leq t \leq T}\left\|\nabla_{x} u(t)\right\|_{\infty} .
$$

Hence $u$ satisfies the following equation,

$$
\left\{\begin{array}{l}
-\partial_{t} u-\nu \Delta u=-H\left(x, \nabla_{x} u^{1}, \mu^{1}\right)+H\left(x, \nabla_{x} u^{2}, \mu^{2}\right), \\
u(T, x)=g\left(x, m^{1}(T)\right)-g\left(x, m^{2}(T)\right) .
\end{array}\right.
$$


The right-hand side of the first line can be estimated in absolute value from above as follows:

$$
\left|H\left(x, \nabla_{x} u^{1}, \mu^{1}\right)-H\left(x, \nabla_{x} u^{2}, \mu^{2}\right)\right| \leq C \sup _{0 \leq t^{\prime} \leq T}\left\|\nabla_{x} u\left(t^{\prime}\right)\right\|_{\infty}
$$

by $\mathbf{T}$, (5.7) and (5.8). Since $u(T, \cdot) \in C^{1+\beta}\left(\mathbb{T}^{d}\right)$, Theorem 6.48 in 34$]$ yields that $u \in C^{\frac{1}{2}+\frac{\beta}{2}, 1+\beta}\left([0, T] \times \mathbb{T}^{d}\right)$ and it satisfies:

$$
\begin{aligned}
\sup _{t \in[0, T]}\left\|\nabla_{x} u(t)\right\|_{\infty} \leq\left\|\nabla_{x}\left(g\left(\cdot, m^{1}(T)\right)-g\left(\cdot, m^{2}(T)\right)\right)\right\|_{\infty} & \\
& +C T^{\frac{\beta}{2}} \sup _{t \in[0, T]}\left(\left\|\nabla_{x} u(t)\right\|_{\infty}+\left\|g\left(\cdot, m^{1}(T)\right)-g\left(\cdot, m^{2}(T)\right)\right\|_{C^{1+\beta}}\right) .
\end{aligned}
$$

This, (2.5) and (5.8) yield,

$$
\sup _{t \in[0, T]}\left\|\nabla_{x} u(t)\right\|_{\infty} \leq C T^{\frac{\beta}{2}} \sup _{t \in[0, T]}\left\|\nabla_{x} u(t)\right\|_{\infty} .
$$

Thus if we suppose furthermore that $T<C^{-\frac{2}{\beta}}$, then $\nabla_{x} u=0$, so $m=0$ by (5.8), then $\mu^{1}=\mu^{2}$ by (5.7), and finally $u^{1}$ and $u^{2}$ solve the same Hamilton-Jacobi-Bellman equation with the same terminal condition, so by uniqueness $u=0$.

Therefore, we proved the uniqueness for $T<T_{1}$ where $T_{1}$ is defined by $T_{1}=\min T_{0},\left(C^{-\frac{2}{\beta}}, C^{-1}\right)$.

\section{6 - ApplicAtions}

Here, we are going to work on $\mathbb{T}^{d}$, while it would be more realistic to work in the whole space $\mathbb{R}^{d}$ for the applications considered below. We would like to recall that the existence results contained in the present work hold for MFGC systems on $\mathbb{R}^{d}$ using the method introduced in [28] to pass from the torus to the whole Euclidean space. Therefore, the conclusions of this section may be adapted to treat the same applications on $\mathbb{R}^{d}$.

\subsection{Exhaustible ressource model with nonpositively correlated ressources}

This model is often referred to as Bertrand and Cournot competition model for exhaustible ressources, introduced in the independent works of Cournot [17] and Bertrand [6]; its mean field game version in dimension one was introduced in [24] and numerically analyzed in [16]; for theoretical results see [8, 22, 27, 23]. We consider a continuum of producers selling exhaustible ressources. The production of a representative agent is $\left(q_{t}\right)_{t \in[0, T]}$; the agents differ in their production capacities $X_{t} \in \mathbb{T}$ (the state variable), that satifies,

$$
d X_{t}=-q_{t} d t+\sqrt{2 \nu} d W_{t},
$$

where $\nu>0$ and $W$ is a Brownian motion. Each producer is selling a different ressource and has her own consumers. However, the ressources are substitutable and any consumer may change her mind and buy from a competitor depending on the degree of competition in the game (which is characterized by $\varepsilon$ in the linear demand case below for instance). Therefore, the selling price per unit of ressource that a producer can make when she sales $q$ units of ressource, depends naturally on $q$ and on the quantity produced by the other agents. The price satisfies a supply-demand relationship, and is given by $P(q, \bar{q})$, where $\bar{q}$ is the aggregate demand which depends on the overall distribution of productions of the agents. A producer tries to maximize her profit, or equivalently to minimize the following quantity,

$$
\mathbb{E}\left[\int_{0}^{T}-P\left(q_{t}, \bar{q}_{t}\right) \cdot q_{t} d t+g\left(X_{T}\right)\right]
$$


where $g$ is a terminal cost which often penalizes the producers who have non-zero production capacities at the end of the game. In the Cournot competition, see [17], a producer is controling her production $q$. Like the MFG version of the Bertrand and Cournot competition introduced in [16], here we consider the Bertrand formulation [6], where an agent directly controls her selling price $\alpha=P(q, \bar{q})$. After inverting the latter equality, the production can be viewed as a function of the price and the mean field. Mathematically this corresponds to writing $q=Q(\alpha, \bar{\alpha})$.

In [16], the authors considered a linear demand system depending on $\bar{q}_{\text {lin }}=\int_{\mathbb{T}} q(x) d m(x)$, and a price satisfying $\alpha=P_{\text {lin }}\left(q, \bar{q}_{\text {lin }}\right)=1-q-\varepsilon \bar{q}_{\text {lin }}$. In this case, the running cost $L^{\text {lin }}$ and its Legendre transform $H^{\text {lin }}$ are defined by

$$
\begin{aligned}
& L^{\operatorname{lin}}(\alpha, \mu)=\alpha^{2}+\frac{\varepsilon}{1+\varepsilon} \alpha \bar{\alpha}-\frac{1}{1+\varepsilon} \alpha, \\
& H^{\operatorname{lin}}(p, \mu)=\frac{1}{4}\left(p+\frac{\varepsilon}{1+\varepsilon} \bar{\alpha}-\frac{1}{1+\varepsilon}\right)^{2},
\end{aligned}
$$

where $\alpha, p \in \mathbb{R}, \mu \in \mathcal{P}(\mathbb{T} \times \mathbb{R})$ and $\bar{\alpha}$ is defined by $\bar{\alpha}=\int_{\mathbb{T} \times \mathbb{R}} \widetilde{\alpha} d \mu(y, \widetilde{\alpha})$. Therefore the system of MFGC has the following form,

$$
\left\{\begin{array}{l}
-\partial_{t} u-\nu \Delta u+\frac{1}{4}\left(\nabla_{x} u+\frac{\varepsilon}{1+\varepsilon} \bar{\alpha}-\frac{1}{1+\varepsilon}\right)^{2}=0 \\
\partial_{t} m-\nu \Delta m-\operatorname{div}\left(\frac{1}{2}\left(\nabla_{x} u+\frac{\varepsilon}{1+\varepsilon} \bar{\alpha}-\frac{1}{1+\varepsilon}\right) m\right)=0 \\
\bar{\alpha}(t)=-\int_{\mathbb{T}} \frac{1}{2}\left(\nabla_{x} u+\frac{\varepsilon}{1+\varepsilon} \bar{\alpha}(t)-\frac{1}{1+\varepsilon}\right) d m(t, x), \\
u(T, x)=g(x) \\
m(0, x)=m_{0}(x)
\end{array}\right.
$$

for $(t, x) \in[0, T] \times \mathbb{T}$. Roughly speaking, $\varepsilon=0$ corresponds to a monopoly in which a producer does not suffer from competition, and she plays as if she was alone in the game. Conversely, $\varepsilon=\infty$ stands for all the producers selling the same ressource and the consumers not having any preference.

Here, Theorem 2.5 d) implies the following existence result.

Proposition 6.1. If $m_{0}$ and $g$ satisfy $\mathbf{A 2}$ and $\mathbf{\mathbf { A 3 }}$, there exists a solution to (6.1) for any $\varepsilon \in(0, \infty)$.

To prove it, we may take $q=2, q_{0}=1, \lambda_{0}=\frac{\varepsilon}{2(1+\varepsilon)}, \lambda_{1}=1$, and $C_{0}=\frac{1}{2}$ in FP1 then we check the assumptions of Theorem 2.5 d). In this case, the inequality in B3 has the form $1<\left(\frac{2+\varepsilon}{1+\varepsilon}\right)^{2}$, and is satisfied for any $\varepsilon \in(0, \infty)$.

Here, the Lagrangian $L^{\text {lin }}$ satisfies a monotonicity assumption, but the latter existence result does not take advantage of it. We refer to [28] for a uniqueness result and an other existence result for the solution to (6.1) using this monotonicity assumption. Generalizations of (6.1) to larger dimensions with more general Hamiltonians and prices are also discussed in [28] under the monotonicty assumption.

In what follows, we provide a simple example of a generalization of (6.1) in which the monotonicity assumption does not hold and the results in [28] do not apply anymore. However, the results in the present work may hold in some cases even without the monotonicity assumption.

Let us consider a model in which every producer sells $d$ different kinds of ressources. The price of each ressource depends on the mean field like in (6.1). Namely, we take $Q=M \bar{\alpha}-\alpha$ which is now a $d$-dimensional vector and where $M \in \mathbb{R}^{d \times d}$ is a given matrix. This leads to the 
following MFGC system,

$$
\left\{\begin{array}{l}
-\partial_{t} u-\nu \Delta u+\frac{1}{4}\left(\nabla_{x} u+M \bar{\alpha}\right)^{2}=f(x, m), \\
\partial_{t} m-\nu \Delta m-\operatorname{div}\left(\frac{1}{2}\left(\nabla_{x} u+M \bar{\alpha}\right) m\right)=0, \\
\bar{\alpha}(t)=-\left(I_{d}+\frac{1}{2} M\right)^{-1} \int_{\mathbb{R}^{d}} \nabla_{x} u(t, x) d m(t, x), \\
u(T, x)=g(x), \\
m(0, x)=m_{0}(x),
\end{array}\right.
$$

Proposition 6.2. Assume A2, A3, that $M$ has an operator norm smaller than 1 , and that $f$ is continuous, and differentiable with respect to $x$ with continuous derivatives. There exists a solution to (6.3).

The proof consists in taking $q=2, q_{0}=1, \lambda_{1}=1, C_{0}=\frac{1}{2}$ in FP1, and $\lambda_{0}=\frac{\|M\|}{2}$, where $\|M\|$ is the operator norm of $M$; and we check the assumptions of Theorem 2.5 d).

The monotonicity assumption discussed in [28] is equivalent to assuming that $M$ is a positive semi-definite matrix. Here, we do not make such an assumpion.

What we have in mind in the latter example is the case where the prices of the different ressources may be negatively correlated, like cars and oil (if the production of cars increases, then the demand for oil also increases and the price of oil rises while the price of cars decreases), or pesticides and medicines, or gold and other raw materiels. To our knowledge, such a generalization of the exhaustible ressource model to negatively correlated ressources is new in the MFG literature.

More generally, we believe that our results hold for the following MFGC system under various different sets of assumptions that we will not detailed here,

$$
\left\{\begin{array}{l}
-\partial_{t} u-\nu \Delta u+H\left(x, \nabla_{x} u+Q(t, x, \mu)\right)=f(t, x, m(t)), \\
\partial_{t} m-\nu \Delta m-\operatorname{div}\left(H_{p}\left(x, \nabla_{x} u+Q(t, x, \mu)\right) m\right)=0, \\
\mu(t)=\left(I_{d},-H_{p}\left(\cdot, \nabla_{x} u(t, \cdot)+Q(t, \cdot, \mu(t))\right) \# m(t)\right. \\
u(T, x)=g(x, m(T)), \\
m(0, x)=m_{0}(x),
\end{array}\right.
$$

where $Q:[0, T] \times \mathbb{T}^{d} \times \mathcal{P}\left(\mathbb{T}^{d} \times \mathbb{R}^{d}\right) \rightarrow \mathbb{R}^{d}$ is a vector characterizing the mean field interactions.

\subsection{Price impact models with bid and ask prices}

The price impact model without bid and ask prices is inspired by the Almgren and Chriss's model [5], and was introduced in the MFG literature in [11] and [15] where existence and uniqueness results are proved when the admissible controls stay in a compact set. Here we consider an extension with bid and ask prices.

We suppose that a continuum of agents are trading an asset, the state of a representative agent is $X_{t}$ the amount of this asset she owns. Her control $\alpha$ is the quantity she buys (if $\alpha \geq 0$ ) or sell (if $\alpha<0$ ). The state space is the one-dimensional torus $\mathbb{T}$, and $X_{t}$ is given by,

$$
d X_{t}=\alpha_{t} d t+\sigma d W_{t}
$$

where $W$ is a Brownian motion, and $\sigma>0$ is a real constant. We define $S_{t}$ as the asking price of the asset, and $\varepsilon(\mu(t))$ as the difference between the bidding and asking prices, where $\mu(t)$ is the law of $\left(X_{t}, \alpha_{t}\right)$. The agent buys at the bidding price $S_{t}+\varepsilon\left(\mu_{t}\right)$, thus her cash is given by

$$
d K_{t}=-\left(\alpha_{t} S_{t}+\alpha_{t} \varepsilon(\mu(t))+\ell\left(\alpha_{t}\right)\right) d t,
$$


where $\ell$ is a differentiable function standing for the transaction cost. The price $S_{t}$ evolves accordingly with the amount of transactions at time $t$, it satisfies the following SDE,

$$
\begin{aligned}
& d S_{t}=A(\mu(t)) d t, \\
& \text { where } A(\mu(t))=\int_{\mathbb{T} \times \mathbb{R}} \ell^{\prime}(\alpha) d \mu(t, x, \alpha),
\end{aligned}
$$

The wealth of a representative agent is given by $V_{t}=V_{0}+X_{t} S_{t}+K_{t}$ and it satisfies the following SDE,

$$
d V_{t}=\left(X_{t} A(\mu(t))-\ell\left(\alpha_{t}\right)-\varepsilon(\mu(t)) \alpha_{t}\right) d t+\sigma S_{t} d W_{t}
$$

The objective function that she will try to maximize is given by,

$$
\mathbb{E}\left[V_{T}-\int_{0}^{T} f\left(X_{t}\right) d t-g\left(X_{T}\right)\right]
$$

where $f$ and $g$ are penalization costs for holding stocks. Here, the Lagrangian and Hamiltonian are given by,

$$
\begin{aligned}
& L^{\mathrm{PI}}(x, \alpha, \mu)=\ell(\alpha)+\alpha \varepsilon(\mu)-x A(\mu), \\
& H^{\mathrm{PI}}(x, p, \mu)=h(p+\varepsilon(\mu))+x A(\mu),
\end{aligned}
$$

for $(x, \alpha, \mu) \in \mathbb{T} \times \mathbb{R} \times \mathcal{P}(\mathbb{T} \times \mathbb{R})$, where $h$ is the Legendre transform of $\ell$.

The linear-quadratic case with $\varepsilon=0$ is treated in [13. Here, taking $\varepsilon=0$ corresponds to assuming that the bidding and asking prices coincide. In this case the optimal control is given by $-h_{p}(p)$ and does not depend explicitely on $\mu$. If $\varepsilon \neq 0$, the optimal control depends explicitely on $\mu$ and $L^{\mathrm{PI}}$ is not separable in $\alpha$ and $\mu$, this prevents us from using the results in [13].

Let us give an example of choices for the functions $\ell$ and $\varepsilon$ under which our result apply and a solution of the MFGC price impact model exists.

Proposition 6.3. Assume $\mathbf{\text { A2 }}$, A3, that $f$ is $C^{1}$, and that $c$ and $\varepsilon$ are respectively given by $\ell(\alpha)=\frac{|\alpha|^{2}}{2}$ and $\varepsilon(\mu)=\widetilde{\varepsilon}\left(\int_{\mathbb{T} \times \mathbb{R}}|\alpha|^{2} d \mu(x, \alpha)\right)^{\frac{1}{2}}$, where $0<\widetilde{\varepsilon}<\frac{1}{2}$. There exists a solution to (1.2) with $H^{P I}$.

This existence result is a consequence of 2.5 c) , where the assumptions are satisfied for $q=q_{0}=2, \lambda_{0}=\varepsilon, \lambda_{1}=\frac{1}{4}$ and $C_{0}=1 \mathrm{in}$ FP1. We would like to insist on the fact that Theorem 2.5 c) provides the existence of solutions for a wild class of Hamiltonian, larger than the one of the latter proposition and which goes beyond the linear-quadratic case.

Let us mention that we would be interested in defining the bidding price by $(1+\widetilde{\varepsilon}) S_{t}$, where $\widetilde{\varepsilon}>0$. The associated MFGC system cannot be using the conclusions of the present work because the mean field interaction at time $t$ would depend not only on $\mu_{t}$ but on $\left(\mu_{s}\right)_{s \in[0, t]}$. However, we believe that existence holds under similar assumptions as here, and we plan to prove it in forthcoming works.

\subsection{First-order flocking model with velocity as controls}

Cucker and Smale proposed a form of Vicseck model in [18] to illustrate the behavior of flocks of birds. This model is of second-order in the sense that the state of an agent is given by a couple $(x, v)$ standing for her position and velocity respectively, and the equation of evolution of her state involves considering her acceleration.

A game version of this model in which an agent controls her acceleration has been introduced in [36], the authors derived a MFG formulation in the infinite horizon case. Here we are interested 
in the finite horizon problem which was studied in [15, 13. This model is still of secondorder. More precisely the state of an agent is given by $\left(X_{t}, V_{t}\right)_{t \in[0, T]}$ respectively her position and velocity, two random processes which satisfy the following system of stochastic differential equations,

$$
\begin{aligned}
d X_{t} & =V_{t} d t, \\
d V_{t} & =a_{t} d t+\sigma d W_{t},
\end{aligned}
$$

where $a_{t}$ is the individual's acceleration vector and her control, $W$ is a $d$-dimentional Brownian motion, and $\sigma \in \mathbb{R}^{d \times d}$ is a positive definite matrix. The cost that a representative agent tries to minimize is given by

$$
\mathbb{E}\left[\int_{0}^{T} \frac{\left|a_{t}\right|^{2}}{2}+\frac{1}{2}\left|\int_{\mathbb{T}^{d}}\left(v-V_{t}^{i}\right) \varphi\left(\left|x-X_{t}^{i}\right|\right) d \mu(t, x, v)\right|^{2}+f\left(X_{t}\right) d t\right],
$$

where $\mu(t) \in \mathcal{P}\left(\mathbb{T}^{d} \times \mathbb{R}^{d}\right)$ is the joint distribution of states and velocities of the agents, $\varphi$ is a $C^{1}$ nonincreasing function, and $f$ is a $C^{1}$ function modeling the spatial preferences of the agents (for instance, we can take $f$ significantly smaller in some areas which corresponds to where the food is).

Here we consider an alternative viewpoint in which an agent directly controls her velocity. This is a first-order model since the state of an agent is now given by a vector of $\mathbb{T}^{d}$, and the acceleration does not appear anymore in the dynamics of a given agent, which is given by

$$
d X_{t}=\alpha_{t} d t+\sigma d W_{t}
$$

Here, the cost that an agent tries to minimize is given by

$$
\mathbb{E}\left[\int_{0}^{T} \frac{\left|\alpha_{t}\right|^{2}}{2}+\frac{1}{2}\left|\int_{\mathbb{T}^{d} \times \mathbb{R}^{d}}\left(\widetilde{\alpha}-\alpha_{t}\right) \varphi\left(\left|x-X_{t}^{i}\right|\right) d \mu(t, x, \widetilde{\alpha})\right|^{2}+f\left(X_{t}\right) d t\right] .
$$

First-order physical models are generally easier to study than second-order models. However the price we paid here to go from a second-order model to a first-order model is to consider a MFGC system instead of a MFG system without interaction through the controls.

If $\mu \in \mathcal{P}\left(\mathbb{T}^{d} \times \mathbb{R}^{d}\right)$ and $m \in \mathcal{P}\left(\mathbb{T}^{d}\right)$ are such that $m$ is the marginal of $\mu$ with respect to $\mathbb{T}^{d}$, we define $A(x, \mu)$ and $Z(x, \mu)$ by,

$$
\left\{\begin{array}{l}
A(x, \mu)=\int_{\mathbb{T}^{d} \times \mathbb{R}^{d}} \widetilde{\alpha} \varphi(|x-y|) d \mu(y, \widetilde{\alpha}), \\
Z(x, \mu)=\int_{\mathbb{T}^{d}} \varphi(|x-y|) d m(y),
\end{array}\right.
$$

for $x \in \mathbb{T}^{d}$. We define the Lagrangian of the first-order flocking model by,

$$
L^{\mathrm{FM}}(x, \alpha, \mu)=\frac{|\alpha|^{2}}{2}+\frac{1}{2}|Z(x, \mu) \alpha-A(x, \mu)|^{2}+f(x),
$$

for $(x, \alpha, \mu) \in \mathbb{T}^{d} \times \mathbb{R}^{d} \times \mathcal{P}\left(\mathbb{T}^{d} \times \mathbb{R}^{d}\right)$, and the Hamiltonian by,

$$
H^{\mathrm{FM}}(x, p, \mu)=\frac{1}{2\left(1+Z(x, \mu)^{2}\right)}\left(|p|^{2}-2 Z(x, \mu) A(x, \mu) \cdot p-|A(x, \mu)|^{2}\right)-f(x),
$$

for $p \in \mathbb{R}^{d}$, such that $H^{\mathrm{FM}}$ is the Legendre's transform of $L^{\mathrm{FM}}$.

Proposition 6.4. Under assumptions $\mathbf{\mathbf { A 2 }}$ and $\mathbf{\mathbf { A 3 }}$, there exists $T_{0}>0$ such that if $T<T_{0}$, there exists a unique solution to (1.2) with $H^{F M}$.

Hereafter, we present an other model for crowd motion which is very similar to the first-order flocking model discussed above. The main difference between these two models is the normalization constants. However, the assumptions and conclusions of this work are more adapted to the following crowd motion model and we can derive more existence results for it. We believe that these results can be adapted to the first-order Cucker-Smale system. 


\subsection{A model of crowd motion}

This model of crowd motion has been numerically studied in [1] in the quadratic case, and has some similarities with the first-order flocking model presented in the previous paragraph. For $(x, \mu) \in \mathbb{T}^{d} \times \mathcal{P}\left(\mathbb{T}^{d} \times \mathbb{R}^{d}\right)$, we define $V(x, \mu)$ and $Z_{q_{0}}(x, \mu)$ by

$$
\left\{\begin{aligned}
V(x, \mu) & =\frac{1}{Z_{q_{0}}(x, \mu)} \int_{\mathbb{T}^{d} \times \mathbb{R}^{d}} \widetilde{\alpha} k(x, y) d \mu(y, \widetilde{\alpha}), \\
Z_{q_{0}}(x, \mu) & =\left(\int_{\mathbb{T}^{d}} k(x, y)^{q_{0}^{\prime}} d m(y)\right)^{\frac{1}{q_{0}^{\prime}}}
\end{aligned}\right.
$$

where $q_{0} \in(1, \infty], q_{0}^{\prime}$ is the conjugate exponent of $q_{0}, k: \mathbb{T}^{d} \times \mathbb{T}^{d} \rightarrow \mathbb{R}_{+}$is a nonnegative $C^{1}$ kernel, and $m \in \mathcal{P}\left(\mathbb{T}^{d}\right)$ is the marginal of $\mu$ with respect to $\mathbb{T}^{d}$. The quantity $V(x, \mu)$ is called the average drift.

The state of a representative agent is given by her position $X_{t} \in \mathbb{T}^{d}$ and she controls her velocity $\alpha_{t}$,

$$
d X_{t}=\alpha_{t} d t+\sqrt{2 \nu} d W_{t}
$$

Her objective is to minimize the cost given by,

$$
\mathbb{E}\left[\int_{0}^{T} \frac{\theta}{a^{\prime}}\left|\alpha_{t}-\tilde{\lambda} V\left(X_{t}, \mu(t)\right)\right|^{a^{\prime}}+\frac{1-\theta}{b^{\prime}}\left|\alpha_{t}\right|^{b^{\prime}}+f\left(X_{t}\right) d t+g\left(X_{T}\right)\right],
$$

where $-1<\tilde{\lambda}<1$ and $0 \leq \theta \leq 1$ are two constants standing for the preference of an individual to have a similar (resp. opposite) control as the mainstream when $\widetilde{\lambda}>0$ (resp. $\widetilde{\lambda}<0$ ), $f$ and $g$ are respectively the running cost and the terminal cost which encode the spatial preferences of the agents, and $a^{\prime}, b^{\prime}>1$ are exponents.

Here, we take $q=\min (a, b)$. In this model we define the Lagrangian by,

$$
L(x, \alpha, \mu)=\frac{\theta}{a^{\prime}}|\alpha-\tilde{\lambda} V(x, \mu)|^{a^{\prime}}+\frac{1-\theta}{b^{\prime}}|\alpha|^{b^{\prime}},
$$

and the Hamiltonian as its Legendre transform. If $a=b=2, H$ is given by

$$
H(x, p, \mu)=\frac{|p|^{2}}{2}-\tilde{\lambda} \theta p \cdot V(x, \mu)-\frac{\widetilde{\lambda}^{2} \theta(1-\theta)}{2}|V(x, \mu)|^{2} .
$$

If $\theta=1, H$ satisfies

$$
H(x, p, \mu)=\frac{1}{a}|p|^{a}-\tilde{\lambda} p \cdot V(x, \mu) .
$$

For other choices of the parameters $a, b$ and $\theta, H$ does not admit an explicit form.

Proposition 6.5. Assume that $g$ and $m_{0}$ satisfy $\mathbf{\mathbf { A 2 }}$ and $\mathbf{\mathbf { A 3 }}$ respectively. There exists a solution to (1.2) where $H$ is the Legendre transform of $L$ given in (6.5), under one of the following assertions,

a) $q_{0} \leq q^{\prime}$ and $a \neq b$,

b) $q_{0} \leq q^{\prime}$ and one of the following assertions is satisfied,

$$
\begin{aligned}
& \text { i) } \theta<\theta_{0}, \\
& \text { ii) } \theta>1-\theta_{0}, \\
& \text { iii) }|\widetilde{\lambda}|<\lambda_{0},
\end{aligned}
$$

where $\theta_{0}, \lambda_{0} \in(0,1)$ are constants coming from Theorem 2.5 c), 
c) $\theta=1$

d) $k(x, y)$ is constant,

e) $T<T_{0}$, where $T_{0}$ is a positive constant coming from Theorem 2.5 e).

Proof. We refer to the appendix, Lemma A.2 for the proof that $H$ satisfies A1,A3, B1,B3, FP1 FP2, and T. The existence results c), d) and e) are direct consequences of Theorem 2.5 c), d) and e) respectively.

We define $\widetilde{L}(\alpha, V)$ by

$$
\widetilde{L}(\alpha, V)=\frac{\theta}{a^{\prime}}|\alpha-\widetilde{\lambda} V|^{a^{\prime}}+\frac{1-\theta}{b^{\prime}}|\alpha|^{b^{\prime}},
$$

for $\alpha, V \in \mathbb{R}^{d}, \widetilde{H}(p, V)$ as the Legendre transform of $\widetilde{L}$ with respect to its first argument, and $\boldsymbol{\alpha}(p, V)$ as the unique control which achieves the maximum in the definition of $\widetilde{H}$ (it is unique because $\widetilde{L}$ is strictly convex with respect to $\alpha$ ).

Proof of $a$ ). Take $V \in \mathbb{R}^{d}$ and $\boldsymbol{\alpha}=\boldsymbol{\alpha}(0, V)$, since $\boldsymbol{\alpha}$ achieves the maximum in the definition of $\widetilde{H}(0, V)$, we know that

$$
0=\theta|\boldsymbol{\alpha}-\tilde{\lambda} V|^{a^{\prime}-2}(\boldsymbol{\alpha}-\tilde{\lambda} V)+(1-\theta)|\boldsymbol{\alpha}|^{b^{\prime}-2} \boldsymbol{\alpha},
$$

which implies

$$
\theta|\boldsymbol{\alpha}-\tilde{\lambda} V|^{a^{\prime}-1}=(1-\theta)|\boldsymbol{\alpha}|^{b^{\prime}-1}
$$

and then

$$
\left(\theta^{a-1}(1-\theta)^{2-a}|\boldsymbol{\alpha}|^{\frac{\left(a^{\prime}-2\right)\left(b^{\prime}-1\right)}{a^{\prime}-1}}+(1-\theta)|\boldsymbol{\alpha}|^{b^{\prime}-2}\right) \boldsymbol{\alpha}=\widetilde{\lambda} \theta^{a-1}(1-\theta)^{2-a}|\boldsymbol{\alpha}|^{\frac{\left(a^{\prime}-2\right)\left(b^{\prime}-1\right)}{a^{\prime}-1}} V .
$$

The two latter equalities yield $\lim _{V \rightarrow+\infty}|\boldsymbol{\alpha}(0, V)|=+\infty$. We make out two cases:

- if $a>b$ then we have $\frac{\left(a^{\prime}-2\right)\left(b^{\prime}-1\right)}{a^{\prime}-1}<b^{\prime}-2$, and $|\boldsymbol{\alpha}|=\underset{+\infty}{o}(|V|)$. Therefore, (66.7) yields

$$
|\boldsymbol{\alpha}|^{b^{\prime}-1-\frac{\left(a^{\prime}-2\right)\left(b^{\prime}-1\right)}{a^{\prime}-1}}=\underset{+\infty}{O}(|V|)
$$

and $b^{\prime}-1-\frac{\left(a^{\prime}-2\right)\left(b^{\prime}-1\right)}{a^{\prime}-1}=\frac{a-1}{b-1}>1$, so we obtain

$$
|\boldsymbol{\alpha}|=\underset{+\infty}{O}\left(|V|^{\frac{b-1}{a-1}}\right)
$$

which yields

$$
\widetilde{H}(0, V)=\underset{+\infty}{O}\left(|V|^{a^{\prime}}\right)+\underset{+\infty}{O}\left(|V|^{\frac{b-1}{a-1} b^{\prime}}\right),
$$

with $a^{\prime}<b^{\prime}$, and $\frac{b-1}{a-1} b^{\prime}<b^{\prime}$, and $b=q$.

- if $a<b$ then we have $\frac{\left(a^{\prime}-2\right)\left(b^{\prime}-1\right)}{a^{\prime}-1}>b^{\prime}-2$, and $\boldsymbol{\alpha}=\tilde{\lambda} V+\underset{+\infty}{o}(|V|)$. Therefore, (6.7) yields

$$
\left(1+\underset{+\infty}{O}\left(|V|^{b^{\prime}-2-\frac{\left(a^{\prime}-2\right)\left(b^{\prime}-1\right)}{a^{\prime}-1}}\right)\right) \boldsymbol{\alpha}=\widetilde{\lambda} V
$$

We notice that $b^{\prime}-2-\frac{\left(a^{\prime}-2\right)\left(b^{\prime}-1\right)}{a^{\prime}-1}=\frac{b^{\prime}-a^{\prime}}{a^{\prime}-1}<0$, and we obtain

$$
\boldsymbol{\alpha}=\widetilde{\lambda} V+\underset{+\infty}{O}\left(|V|^{1+\frac{b^{\prime}-a^{\prime}}{a^{\prime}-1}}\right)=\tilde{\lambda} V+\underset{+\infty}{O}\left(|V|^{\frac{a-1}{b-1}}\right)
$$


This implies

$$
\widetilde{H}(0, V)=\underset{+\infty}{O}\left(|V|^{\frac{b^{\prime}-1}{a^{\prime}-1} a^{\prime}}\right),+\underset{+\infty}{O}\left(|V|^{b^{\prime}}\right),
$$

with $b^{\prime}<a^{\prime}$, and $\frac{a-1}{b-1} a^{\prime}<a^{\prime}$, and $a=q$.

We conclude by (A.1) and Theorem 2.5 a).

Proof of b)

Here, we assume that $a=b$ since the case $a \neq b$ is addressed in a)

Take $V \in \mathbb{R}^{d}$, and $\boldsymbol{\alpha}=\boldsymbol{\alpha}(0, V)$. In this case, $\widetilde{H}(0, V)$ admits an explicit form given by

$$
\widetilde{H}(0, V)=-\frac{|\widetilde{\lambda}|^{a^{\prime}}}{a^{\prime}} \frac{\theta(1-\theta)^{a}+(1-\theta) \theta^{a}}{\left((1-\theta)^{a-1}+\theta^{a-1}\right)^{a^{\prime}}}|V|^{a^{\prime}} .
$$

Therefore, taking $\widetilde{\lambda}, \theta$ or $(1-\theta)$ small enough allows one to conclude by (A.1) and Theorem 2.5 b).

Acknowledgements. I wish to express my gratitude to Y. Achdou and P. Cardaliaguet for technical advices, insightful comments and corrections. The work was supported by the ANR project MFG ANR-16-CE40-0015-01.

\section{6 - REFERENCES}

[1] Y Achdou and Z Kobeissi. Mean field games of controls: Finite difference approximations, 2020 .

[2] Yves Achdou. Finite difference methods for mean field games. In Hamilton-Jacobi equations: approximations, numerical analysis and applications, volume 2074 of Lecture Notes in Math., pages 1-47. Springer, Heidelberg, 2013.

[3] Yves Achdou and Jean-Michel Lasry. Mean field games for modeling crowd motion. In Contributions to partial differential equations and applications, volume 47 of Comput. Methods Appl. Sci., pages 17-42. Springer, Cham, 2019.

[4] Clémence Alasseur, Imen Ben Taher, and Anis Matoussi. An extended mean field game for storage in smart grids. J. Optim. Theory Appl., 184(2):644-670, 2020.

[5] Robert Almgren and Neil A. Chriss. Optimal execution of portfolio trans-actions. 2000.

[6] Joseph Bertrand. Théorie mathématiques de la richesse sociale. Journal des Savants, 67:499$508,1883$.

[7] Charles Bertucci, Jean-Michel Lasry, and Pierre-Louis Lions. Some remarks on mean field games. Comm. Partial Differential Equations, 44(3):205-227, 2019.

[8] Frédéric J. Bonnans, Saeed Hadikhanloo, and Laurent Pfeiffer. Schauder Estimates for a Class of Potential Mean Field Games of Controls. arXiv e-prints, page arXiv:1902.05461, Feb 2019.

[9] P Cardaliaguet. Notes on mean field games. Unpublished notes, 2013. https://www.ceremade.dauphine.fr/ cardaliaguet/.

[10] Pierre Cardaliaguet, François Delarue, Jean-Michel Lasry, and Pierre-Louis Lions. The master equation and the convergence problem in mean field games, volume 201 of Annals of Mathematics Studies. Princeton University Press, Princeton, NJ, 2019. 
[11] Pierre Cardaliaguet and Charles-Albert Lehalle. Mean field game of controls and an application to trade crowding. Math. Financ. Econ., 12(3):335-363, 2018.

[12] René Carmona and François Delarue. Probabilistic analysis of mean-field games. SIAM J. Control Optim., 51(4):2705-2734, 2013.

[13] René Carmona and François Delarue. Probabilistic theory of mean field games with applications. I, volume 83 of Probability Theory and Stochastic Modelling. Springer, Cham, 2018. Mean field FBSDEs, control, and games.

[14] René Carmona and François Delarue. Probabilistic theory of mean field games with applications. II, volume 84 of Probability Theory and Stochastic Modelling. Springer, Cham, 2018. Mean field games with common noise and master equations.

[15] René Carmona and Daniel Lacker. A probabilistic weak formulation of mean field games and applications. Ann. Appl. Probab., 25(3):1189-1231, 2015.

[16] Patrick Chan and Ronnie Sircar. Bertrand and Cournot mean field games. Appl. Math. Optim., 71(3):533-569, 2015.

[17] A.A. Cournot. Recherches sur les principes mathématiques de la théorie des richesses. 1838.

[18] Felipe Cucker and Steve Smale. Emergent behavior in flocks. IEEE Trans. Automat. Control, 52(5):852-862, 2007.

[19] David Gilbarg and Neil S. Trudinger. Elliptic partial differential equations of second order. Classics in Mathematics. Springer-Verlag, Berlin, 2001. Reprint of the 1998 edition.

[20] D. A. Gomes and V. K. Voskanyan. Extended mean field games. Izv. Nats. Akad. Nauk Armenii Mat., 48(2):63-76, 2013.

[21] Diogo A. Gomes, Stefania Patrizi, and Vardan Voskanyan. On the existence of classical solutions for stationary extended mean field games. Nonlinear Anal., 99:49-79, 2014.

[22] P. Jameson Graber and Alain Bensoussan. Existence and uniqueness of solutions for Bertrand and Cournot mean field games. Appl. Math. Optim., 77(1):47-71, 2018.

[23] Philip Jameson Graber and Charafeddine Mouzouni. Variational mean field games for market competition. In PDE models for multi-agent phenomena, volume 28 of Springer INdAM Ser., pages 93-114. Springer, Cham, 2018.

[24] Olivier Guéant, Jean-Michel Lasry, and Pierre-Louis Lions. Mean field games and applications. In Paris-Princeton Lectures on Mathematical Finance 2010, volume 2003 of Lecture Notes in Math., pages 205-266. Springer, Berlin, 2011.

[25] Minyi Huang, Peter E. Caines, and Roland P. Malhamé. Large-population cost-coupled LQG problems with nonuniform agents: individual-mass behavior and decentralized $\epsilon$-Nash equilibria. IEEE Trans. Automat. Control, 52(9):1560-1571, 2007.

[26] Minyi Huang, Roland P. Malhamé, and Peter E. Caines. Large population stochastic dynamic games: closed-loop McKean-Vlasov systems and the Nash certainty equivalence principle. Commun. Inf. Syst., 6(3):221-251, 2006.

[27] Philip Jameson Graber and Charafeddine Mouzouni. On Mean Field Games models for exhaustible commodities trade. ESAIM Control Optim. Calc. Var., 26, 2020.

[28] Z Kobeissi. Mean field games with monotonous interactions through the law of states and controls of the agents, 2020. 
[29] Daniel Lacker. Mean field games via controlled martingale problems: existence of Markovian equilibria. Stochastic Process. Appl., 125(7):2856-2894, 2015.

[30] O. A. Ladyzenskaja, V. A. Solonnikov, and N. N. Ural'ceva. Linear and quasilinear equations of parabolic type. Translated from the Russian by S. Smith. Translations of Mathematical Monographs, Vol. 23. American Mathematical Society, Providence, R.I., 1968.

[31] Jean-Michel Lasry and Pierre-Louis Lions. Jeux à champ moyen. I. Le cas stationnaire. $C$. R. Math. Acad. Sci. Paris, 343(9):619-625, 2006.

[32] Jean-Michel Lasry and Pierre-Louis Lions. Jeux à champ moyen. II. Horizon fini et contrôle optimal. C. R. Math. Acad. Sci. Paris, 343(10):679-684, 2006.

[33] Jean-Michel Lasry and Pierre-Louis Lions. Mean field games. Jpn. J. Math., 2(1):229-260, 2007.

[34] Gary M. Lieberman. Second order parabolic differential equations. World Scientific Publishing Co., Inc., River Edge, NJ, 1996.

[35] Pierre-Louis Lions. Théorie des jeux à champs moyen. video lecture series at Collège de France, 2011-2019. https://www.college-de-france.fr/site/pierre-louis-lions/index.htm.

[36] M. Nourian, P. E. Caines, and R. P. Malhamé. Synthesis of cucker-smale type flocking via mean field stochastic control theory: Nash equilibria. In 2010 48th Annual Allerton Conference on Communication, Control, and Computing (Allerton), pages 814-819, Sep. 2010.

\section{A - VerificAtion of the ASSUmptions FOR THE MODEL OF CROWD MOTION}

We start by establishing some properties of the function $V$ in the following lemma.

Lemma A.1. The function $V$ is $C^{1}$ with respect to $x$ and it satisfies

$$
\|V(\cdot, \mu)\|_{\infty} \leq \Lambda_{q_{0}}(\mu),
$$

where $\mu \in \mathcal{P}\left(\mathbb{T}^{d} \times \mathbb{R}^{d}\right)$.

For $m \in \mathcal{P}\left(\mathbb{T}^{d}\right)$ and $\mu^{1}, \mu^{2} \in \mathcal{P}_{m}\left(\mathbb{T}^{d} \times \mathbb{R}^{d}\right)$, the following inequality is satisfied,

$$
\left\|V\left(\cdot, \mu^{1}\right)-V\left(\cdot, \mu^{2}\right)\right\|_{\infty} \leq\left\|\alpha^{\mu^{1}}-\alpha^{\mu^{2}}\right\|_{L^{q_{0}}(m)} .
$$

For $R>0$, there exists $C_{R}>0$ a constant such that,

$$
\left\|V\left(\cdot, \mu^{1}\right)-V\left(\cdot, \mu^{2}\right)\right\|_{\infty} \leq C_{R}\left(\left\|\alpha^{\mu^{1}}-\alpha^{\mu^{2}}\right\|_{\infty}+\left\|m^{1}-m^{2}\right\|_{\infty}\right)
$$

for $\left(m^{i}, \mu^{i}\right)$ such that $m^{i} \in \mathcal{P}\left(\mathbb{T}^{d}\right) \cap C^{0}\left(\mathbb{T}^{d}\right)$ with $m^{i} \geq R^{-1}, \mu^{i} \in \mathcal{P}\left(\mathbb{T}^{d} \times \mathbb{R}^{d}\right)$ with $\alpha^{\mu^{i}} \in$ $C^{0}\left(\mathbb{T}^{d} \times \mathbb{R}^{d}\right)$ and $\left\|\alpha^{\mu^{i}}\right\|_{\infty} \leq R, i=1,2$.

Proof. The function $V$ has at least the same regularity as $k$ with respect to the state variable since $V$ is the convolution product of $k$ with a probability measure. Then (A.1) and (A.2) are 
straightforward using Hölder inequality. Let us take the same notation as in (A.3), for $x \in \mathbb{T}^{d}$ we get

$$
\begin{aligned}
& \left|V\left(x, \mu^{1}\right)-V\left(x, \mu^{2}\right)\right| \leq \frac{1}{Z_{q_{0}}\left(x, \mu^{1}\right)} \int_{\mathbb{T}^{d}} k(x, y)\left|\alpha^{\mu^{1}}(y)-\alpha^{\mu^{2}}(y)\right| d m^{1}(y) \\
& +\frac{1}{Z_{q_{0}}\left(x, \mu^{1}\right)} \int_{\mathbb{T}^{d}} k(x, y)\left|\alpha^{\mu^{2}}(y)\right|\left|m^{1}(y)-m^{2}(y)\right| d y \\
& +\left|\frac{1}{Z_{q_{0}}\left(x, \mu^{1}\right)}-\frac{1}{Z_{q_{0}}\left(x, \mu^{2}\right)}\right| \int_{\mathbb{T}^{d}} k(x, y)\left|\alpha^{\mu^{2}}(y)\right| d m^{2}(y) \\
& \leq\left\|\alpha^{\mu^{1}}-\alpha^{\mu^{2}}\right\|_{\infty}+\frac{1}{Z_{q_{0}}\left(x, \mu^{1}\right)} \int_{\mathbb{T}^{d}} k(x, y) d y\left\|\alpha^{\mu^{2}}\right\|_{\infty}\left\|m^{1}-m^{2}\right\|_{\infty} \\
& +\frac{1}{Z_{q_{0}}\left(x, \mu^{1}\right)}\left\|\alpha^{\mu^{2}}\right\|_{L_{q_{0}\left(m^{2}\right)}}\left|Z_{q_{0}}\left(x, \mu^{1}\right)-Z_{q_{0}}\left(x, \mu^{2}\right)\right| .
\end{aligned}
$$

Moreover, we know that $Z_{q_{0}}\left(x, \mu^{1}\right) \geq R^{-\frac{1}{q_{0}^{\prime}}}\left(\int_{\mathbb{T}^{d}} k(0, y)^{q_{0}^{\prime}} d y\right)^{\frac{1}{q_{0}^{\prime}}}>0$ where the right-hand side does not depend on $x$, and

$$
\begin{aligned}
\left|Z_{q_{0}}\left(x, \mu^{1}\right)-Z_{q_{0}}\left(x, \mu^{2}\right)\right| & \leq \max _{i=1,2}\left(\frac{1}{q_{0}^{\prime}}\left(Z_{q_{0}}\left(x, \mu^{i}\right)^{q_{0}^{\prime}}\right)^{\frac{1}{q_{0}^{\prime}}-1}\right)\left|Z_{q_{0}}\left(x, \mu^{1}\right)^{q_{0}^{\prime}}-Z_{q_{0}}\left(x, \mu^{2}\right)^{q_{0}^{\prime}}\right| \\
& \leq \frac{1}{q_{0}^{\prime}}\left(\min _{i=1,2} Z_{q_{0}}\left(x, \mu^{i}\right)\right)^{1-q_{0}^{\prime}} \int_{\mathbb{T}^{d}} k(0, y)^{q_{0}^{\prime}} d y\left\|m^{1}-m^{2}\right\|_{\infty} \\
& \leq \frac{1}{q_{0}^{\prime}} R^{\frac{1}{q_{0}}}\left(\int_{\mathbb{T}^{d}} k(0, y)^{q_{0}^{\prime}} d y\right)^{\frac{1}{q_{0}^{\prime}}}\left\|m^{1}-m^{2}\right\|_{\infty} .
\end{aligned}
$$

The latter two chains of inequalities imply $(\underline{\mathrm{A} .3})$ with $C_{R}=1+R^{1+\frac{1}{q_{0}^{\prime}}}+\frac{1}{q_{0}^{\prime}} R^{2}$.

Here, we assume $\theta \in(0,1)$. Indeed, $H$ admits an explicit form when $\theta=0$ or $\theta=1$, then checking A1, A3, B1, B3, FP1,FP2, and Tis straightforward.

Lemma A.2. Assumptions A1, B1,B3, FP1, FP2, and $\mathbf{T}$ are satisfied when $L$ is defined in (6.5).

Proof. We define $\widetilde{L}, \widetilde{H}$ and $\boldsymbol{\alpha}$ as in the proof of 6.5 .

Checking A1, B1 and B2.

The Legendre transform of a function is convex, therefore $H$ is convex with respect to $p$. Since $L$ is strictly convex, $H$ is differentiable with respect to $p$. Moreover, $\boldsymbol{\alpha}=-H_{p}$ thus $H_{p}$ is continuous by the Maximum theorem. Then $H(x, p, \mu)=p \cdot H_{p}(x, p, \mu)-L\left(x,-H_{p}(x, p, \mu), \mu\right)$, so $H$ is continuous. Finally, $H$ is differentiable with respect to $x$ by the envelop theorem and

$$
H_{x}(x, p, \mu)=-L_{x}\left(x,-H_{p}(x, p, \mu), \mu\right),
$$

for $(x, p, \mu) \in \mathbb{T}^{d} \times \mathbb{R}^{d} \times \mathcal{P}\left(\mathbb{T}^{d} \times \mathbb{R}^{d}\right)$.

Using the growth properties of $L$, we can prove that there exists $C_{0}>0$ such that

$$
\begin{aligned}
\left|H_{p}(t, x, p, \mu)\right| & \leq C_{0}\left(1+|p|^{q-1}+\Lambda_{q^{\prime}}(\mu)\right), \\
|H(t, x, p, \mu)| & \leq C_{0}\left(1+|p|^{q}+\Lambda_{q^{\prime}}(\mu)^{q^{\prime}}\right), \\
\left|H_{x}(t, x, p, \mu)\right| & \leq C_{0}\left(1+|p|^{q}+\Lambda_{q^{\prime}}(\mu)^{q^{\prime}}\right),
\end{aligned}
$$

for any $(x, p, \mu) \in \mathbb{T}^{d} \times \mathbb{R}^{d} \times \mathcal{P}\left(\mathbb{R}^{d} \times \mathbb{R}^{d}\right)$. We refer to [28] Lemma 2.5 for a complete proof. 
One may prove that the function $h: z \in \mathbb{R}^{d} \mapsto|z|^{a^{\prime}} \in \mathbb{R}$ satisfies $h(z)-h(y)-\nabla h(y) \cdot(y-x) \geq$ $C_{R}^{-1}|y-z|^{\max \left(a^{\prime}, 2\right)}$ for $y, z \in \mathbb{R}^{d}$ such that $|y| \leq R,|z| \leq R$, where $C_{R}>0$ is a constant. This implies that for $R>0$ there exists $C_{R}>0$ a constant such that $L$ satisfies

$$
L\left(x, \alpha^{2}, \mu\right)-L\left(x, \alpha^{1}, \mu\right)-\left(\alpha^{2}-\alpha^{1}\right) \cdot L_{\alpha}\left(x, \alpha^{1}, \mu\right) \geq C_{R}^{-1}\left|\alpha^{2}-\alpha^{1}\right|^{\max \left(q^{\prime}, 2\right)},
$$

for $\left(\alpha^{1}, \alpha^{2}, \mu\right) \in \mathbb{R}^{d} \times \mathbb{R}^{d} \times \mathcal{P}_{\infty}\left(\mathbb{T}^{d} \times \mathbb{R}^{d}\right)$, such that $\left|\alpha^{i}\right| \leq R$ and $\Lambda_{q_{0}}(\mu) \leq R$. This implies

$$
\left(\alpha^{2}-\alpha^{1}\right) \cdot\left(L_{\alpha}\left(x, \alpha^{2}, \mu\right)-L_{\alpha}\left(x, \alpha^{1}, \mu\right)\right) \geq 2 C_{R}^{-1}\left|\alpha^{2}-\alpha^{1}\right|^{\max \left(q^{\prime}, 2\right)} .
$$

Take $p^{i} \in \mathbb{R}^{d}$ and $\alpha^{i}=-H_{p}\left(x, p^{i}, \mu\right), i=1,2$. Recalling the conjugacy relation $p^{i}=$ $-L_{\alpha}\left(x, \alpha^{i}, \mu\right)$ we obtain that $H_{p}$ is locally Hölder continuous with respect to $p$.

Checking B3.

Take $(p, V) \in \mathbb{R}^{2 d}$ and $\boldsymbol{\alpha}=\boldsymbol{\alpha}(p, V)$, the optimal control $\boldsymbol{\alpha}$ satisfies

$$
p=-D_{\alpha} \widetilde{L}(\boldsymbol{\alpha}, V)=-\theta|\boldsymbol{\alpha}-\widetilde{\lambda} V|^{a^{\prime}-2}(\boldsymbol{\alpha}-\widetilde{\lambda} V)-(1-\theta)|\boldsymbol{\alpha}|^{b^{\prime}-2} \boldsymbol{\alpha},
$$

If $(p, V) \neq(0,0)$, this implies

$$
\boldsymbol{\alpha}=\frac{-p+\widetilde{\lambda} \theta|\boldsymbol{\alpha}-\widetilde{\lambda} V|^{a^{\prime}-2} V}{\theta|\boldsymbol{\alpha}-\widetilde{\lambda} V|^{a^{\prime}-2}+(1-\theta)|\boldsymbol{\alpha}|^{b^{\prime}-2}},
$$

and

$$
\boldsymbol{\alpha}-\widetilde{\lambda} V=\frac{-p+\widetilde{\lambda}(1-\theta)|\boldsymbol{\alpha}|^{b^{\prime}-2} V}{\theta|\boldsymbol{\alpha}-\widetilde{\lambda} V|^{a^{\prime}-2}+(1-\theta)|\boldsymbol{\alpha}|^{b^{\prime}-2}} .
$$

From (A.8), we deduce that

$$
\theta|\boldsymbol{\alpha}-\tilde{\lambda} V|^{a^{\prime}-1} \geq \frac{1}{2}|p|, \text { or }(1-\theta)|\boldsymbol{\alpha}|^{b^{\prime}-1} \geq \frac{1}{2}|p| .
$$

We recall that $\boldsymbol{\alpha}=-H_{p}(p, V)$, hence

$$
\begin{aligned}
\widetilde{H}_{p}(p, V) \cdot p-\widetilde{H}(p, V) & =\widetilde{L}(\boldsymbol{\alpha}, V) \\
& =\frac{\theta}{a^{\prime}}|\boldsymbol{\alpha}-\widetilde{\lambda} V|^{a^{\prime}}+\frac{1-\theta}{b^{\prime}}|\boldsymbol{\alpha}|^{b^{\prime}}, \\
& \geq \min \left(\frac{|p|^{a}}{2^{a} a^{\prime} \theta^{a-1}}, \frac{|p|^{b}}{2^{b} b^{\prime}(1-\theta)^{b-1}}\right),
\end{aligned}
$$

which implies B3.

Proof that $\boldsymbol{\alpha}$ is differentiable with respect to $V$ at $(0,0)$.

Take $V \in \mathbb{R}^{d}$ that will eventually tend to 0 and $\boldsymbol{\alpha}=\boldsymbol{\alpha}(0, V)$. From (A.8) we obtain

$$
0=\theta|\boldsymbol{\alpha}-\tilde{\lambda} V|^{a^{\prime}-2}(\boldsymbol{\alpha}-\tilde{\lambda} V)+(1-\theta)|\boldsymbol{\alpha}|^{b^{\prime}-2} \boldsymbol{\alpha},
$$

Let us recall inequalities (6.6) and (6.7).

- if $a>b$ then $\frac{\left(a^{\prime}-2\right)\left(b^{\prime}-1\right)}{a^{\prime}-1}<b^{\prime}-2$, and we obtain the following expansion as $|V|$ tends to 0 ,

$$
\boldsymbol{\alpha}=\tilde{\lambda} V+o(|V|)
$$

- if $a=b$ we obtain,

$$
\boldsymbol{\alpha}=\widetilde{\lambda} \frac{\theta^{a-1}}{\theta^{a-1}+(1-\theta)^{a-1}} V
$$


- if $a<b$ then $\frac{\left(a^{\prime}-2\right)\left(b^{\prime}-1\right)}{a^{\prime}-1}>b^{\prime}-2$, and we obtain the following estimate as $|V|$ tends to 0 ,

$$
\boldsymbol{\alpha}=o(|V|)
$$

Therefore the derivatives of $\boldsymbol{\alpha}$ with respect to $V$ in any of the above three cases are:

$$
D_{V} \boldsymbol{\alpha}(0,0)= \begin{cases}\tilde{\lambda} I_{d} & \text { if } b<a \\ \tilde{\lambda} \frac{\theta^{a-1}}{\theta^{a-1}+(1-\theta)^{a-1}} I_{d} & \text { if } b=a \\ 0 & \text { if } b>a .\end{cases}
$$

Proof that the operator norm of $D_{V} \boldsymbol{\alpha}=\left(\partial_{V^{j}} \boldsymbol{\alpha}^{i}\right)_{1 \leq i, j \leq d} \in \mathbb{R}^{d \times d}$ is not larger than $\lambda$.

Here, the norm of a square matrix $A \in \mathbb{R}^{d \times d}$ is defined by $\|A\|=\sup _{X \neq 0} \frac{|A X|}{|X|}$. Let us introduce

$$
\begin{array}{ll}
v_{1}=\mathbf{1}_{\boldsymbol{\alpha}-\tilde{\lambda} V \neq 0} \frac{\boldsymbol{\alpha}-\tilde{\lambda} V}{|\boldsymbol{\alpha}-\tilde{\lambda} V|}, & B=I_{d}+\left(a^{\prime}-2\right) v_{1} v_{1}^{T}, \\
v_{2}=\mathbf{1}_{\boldsymbol{\alpha} \neq 0} \frac{\boldsymbol{\alpha}}{|\boldsymbol{\alpha}|}, & C=I_{d}+\left(b^{\prime}-2\right) v_{2} v_{2}^{T} .
\end{array}
$$

We recall that if $v_{i} \neq 0$, then $v_{i} v_{i}^{T}$ is the orthogonal projection onto $\mathbb{R} v_{i}$ for $i=1,2$.

If $\boldsymbol{\alpha}=\widetilde{\lambda} V=0$ then $(p, V)=(0,0)$, we see on (A.12) that $D_{V} \boldsymbol{\alpha}$ is a positive semi-definite matrix with eigenvalues in $[-\lambda, \lambda]$. Therefore, we can now assume that $(\boldsymbol{\alpha}, V) \neq(0,0)$.

Let us assume temporarily that $a^{\prime} \neq 2, b^{\prime} \neq 2, \boldsymbol{\alpha}-\widetilde{\lambda} V \neq 0, \boldsymbol{\alpha} \neq 0$. Then we differentiate the $i$-th component of (A.8) with respect to $V^{j}$,

$$
\begin{aligned}
& 0=\theta|\boldsymbol{\alpha}-\tilde{\lambda} V|^{a^{\prime}-2}\left(\partial_{V^{j}} \boldsymbol{\alpha}^{i}-\tilde{\lambda} \delta_{i, j}\right) \\
&+\theta\left(a^{\prime}-2\right)|\boldsymbol{\alpha}-\tilde{\lambda} V|^{a^{\prime}-4} \sum_{k=1}^{d}\left(\partial_{V^{j}} \boldsymbol{\alpha}^{k}-\tilde{\lambda} \delta_{k, j}\right)\left(\boldsymbol{\alpha}^{i}-\tilde{\lambda} V^{i}\right)\left(\boldsymbol{\alpha}^{k}-\tilde{\lambda} V^{k}\right) \\
& \quad+(1-\theta)|\boldsymbol{\alpha}|^{b^{\prime}-2} \partial_{V^{j}} \alpha^{i}+(1-\theta)\left(b^{\prime}-2\right)|\boldsymbol{\alpha}|^{b^{\prime}-4} \sum_{k=1}^{d} \partial_{V^{j}} \boldsymbol{\alpha}^{k} \boldsymbol{\alpha}^{i} \boldsymbol{\alpha}^{k} .
\end{aligned}
$$

This implies

$$
0=\theta|\boldsymbol{\alpha}-\tilde{\lambda} V|^{a^{\prime}-2} B\left(D_{V} \boldsymbol{\alpha}-\tilde{\lambda} I_{d}\right)+(1-\theta)|\alpha|^{b^{\prime}-2} C D_{V} \boldsymbol{\alpha}
$$

and thus

$$
D_{V} \boldsymbol{\alpha}=\tilde{\lambda}\left[I_{d}+\frac{(1-\theta)|\boldsymbol{\alpha}|^{b^{\prime}-2}}{\theta|\boldsymbol{\alpha}-\widetilde{\lambda} V|^{a^{\prime}-2}} B^{-1} C\right]^{-1}
$$

We can check that this last equation holds in the general case for any $(\boldsymbol{\alpha}, V) \neq(0,0), a^{\prime}, b^{\prime}$.

- If $\left(a^{\prime}-2\right) v_{1}=0$ (i.e. $\left.B=I_{d}\right)$ or $\left(b^{\prime}-2\right) v_{2}=0$ (i.e. $C=I_{d}$ ), then (A.13) yields that $D_{V} \boldsymbol{\alpha}$ is a positive definite matrix with eigenvalues in $(-\lambda, \lambda)$.

- If $\left(a^{\prime}-2\right) v_{1} \neq 0,\left(b^{\prime}-2\right) v_{2} \neq 0$ and $v_{1}, v_{2}$ are aligned, Then $B$ and $C$ commute and $B^{-1} C$ is a positive definite matrix. Then (A.13) yields that $D_{V} \boldsymbol{\alpha}$ is a positive definite matrix with eigenvalues in $(-\lambda, \lambda)$. 
- The last case consists of assuming that $\left(a^{\prime}-2\right) v_{1} \neq 0,\left(b^{\prime}-2\right) v_{2} \neq 0$, and $v_{1}, v_{2}$ are linearly independent. We define $k$ by $k=\frac{(1-\theta)|\boldsymbol{\alpha}|^{b^{\prime}-2}}{\theta|\boldsymbol{\alpha}-\widetilde{\lambda} V|^{a^{\prime}-2}}>0$. The two orthogonal subspaces $\operatorname{Span}\left(v_{1}, v_{2}\right)$ and $\left\{v_{1}, v_{2}\right\}^{\perp}$ are stable by $D_{V} \boldsymbol{\alpha}, B, C$. The restriction of $D_{V} \boldsymbol{\alpha}$ to $\left\{v_{1}, v_{2}\right\}^{\perp}$ is positive definite with eigenvalues in $(-\lambda, \lambda)$.

Let us denote by $\widetilde{A}, \widetilde{B}, \widetilde{C} \in \mathcal{M}_{2 \times 2}(\mathbb{R})$ respectively the restriction of $D_{V} \boldsymbol{\alpha}, B$ and $C$ to $\operatorname{Span}\left(v_{1}, v_{2}\right)$. We notice that

$$
\widetilde{B}^{-1}=I_{d}+\left(\left(a^{\prime}-1\right)^{-1}-1\right) v_{1} v_{1}^{\perp},
$$

thus the eigenvalues of $\widetilde{B}^{-1}$ are 1 and $\left(a^{\prime}-1\right)^{-1} \leq 1$ since $a^{\prime} \geq 2$. The eigenvalues of $\widetilde{C}$ are 1 and $\left(b^{\prime}-1\right) \geq 1$. Lemma A.3 below yields that $M=\left(I_{d}+k \widetilde{B}^{-1} \widetilde{C}\right)\left(I_{d}+k \widetilde{C} \widetilde{B}^{-1}\right)$ is a positive definite matrix with eigenvalues not smaller than 1 . This implies

$$
\begin{aligned}
\|\widetilde{A} X\|^{2} & =\lambda^{2}\left\langle M^{-1} X, X\right\rangle \\
& \leq \lambda^{2}\|X\|^{2} .
\end{aligned}
$$

This concludes the proof that the norm of $D_{V} \boldsymbol{\alpha}$ is not larger than $\lambda$.

\section{Proof of FP2.}

Take $\left(p, V^{1}, V^{2}\right) \in \mathbb{R}^{3 d}$ and $\boldsymbol{\alpha}^{i}=-\widetilde{H}_{p}\left(p, V^{i}\right), i=1,2$, then

$$
\begin{aligned}
\left|\widetilde{H}_{p}\left(p, V_{1}\right)-\widetilde{H}_{p}\left(p, V_{2}\right)\right| & \leq \sup _{s \in[0,1]}\left\{\left\|D_{V} \boldsymbol{\alpha}\left(p, s V_{1}+(1-s) V_{2}\right)\right\|\right\}\left|V^{1}-V^{2}\right| \\
& \leq \lambda\left|V^{1}-V^{2}\right| .
\end{aligned}
$$

Combining the latter inequality and (A.2), we conclude that FP2 is satisfied.

Proof of FP1.

Let $(p, V) \in \mathbb{R}^{2 d}$, we take $\boldsymbol{\alpha}=-H_{p}(p, V)$.

- We suppose $b^{\prime} \geq a^{\prime}$, we make out two cases: the first case is when $|\boldsymbol{\alpha}| \leq|p|^{b-1}$; the second case is when $|\boldsymbol{\alpha}|>|p|^{b-1}=|p|^{\frac{1}{b^{\prime}-1}}$ which implies

$$
\begin{aligned}
|\boldsymbol{\alpha}| & \leq\left|\frac{-p+\widetilde{\lambda} \theta|\boldsymbol{\alpha}-\tilde{\lambda} V|^{a^{\prime}-2} V}{\theta|\boldsymbol{\alpha}-\widetilde{\lambda} V|^{a^{\prime}-2}+(1-\theta)|\boldsymbol{\alpha}|^{b^{\prime}-2}}\right| \\
& \leq \frac{|p|}{(1-\theta)|\boldsymbol{\alpha}|^{b^{\prime}-2}}+\lambda|V| \\
& \leq(1-\theta)^{-1}|p|^{1-\frac{b^{\prime}-2}{b^{\prime}-1}}+\lambda|V|,
\end{aligned}
$$

using (A.9). We recall that $1-\frac{b^{\prime}-2}{b^{\prime}-1}=b-1$, hence

$$
\left|\widetilde{H}_{p}(p, V)\right|=|\boldsymbol{\alpha}| \leq(1-\theta)^{-1}|p|^{b-1}+\lambda|V| \text {. }
$$

- We suppose that $b^{\prime}<a^{\prime}$, we make out two cases: the first case is when $|\boldsymbol{\alpha}-\tilde{\lambda} V| \leq|p|^{a-1}$; the second case is when $|\boldsymbol{\alpha}-\tilde{\lambda} V|>|p|^{\frac{1}{a^{\prime}-1}}$ which implies

$$
\begin{aligned}
|\boldsymbol{\alpha}| & \leq\left|\frac{-p+\widetilde{\lambda} \theta|\boldsymbol{\alpha}-\widetilde{\lambda} V|^{a^{\prime}-2} V}{\theta|\boldsymbol{\alpha}-\widetilde{\lambda} V|^{a^{\prime}-2}+(1-\theta)|\boldsymbol{\alpha}|^{b^{\prime}-2}}\right| \\
& \leq \frac{|p|}{\theta|\boldsymbol{\alpha}-\widetilde{\lambda} V|^{a^{\prime}-2}}+\lambda|V| \\
& \leq \theta^{-1}|p|^{1-\frac{a^{\prime}-2}{a^{\prime}-1}}+\lambda|V|,
\end{aligned}
$$


where we used (A.9). From the equality $1-\frac{a^{\prime}-2}{a^{\prime}-1}=a-1$, we deduce

$$
\left|H_{p}(p, V)\right|=|\boldsymbol{\alpha}| \leq \theta^{-1}|p|^{a-1}+\lambda|V|
$$

This concludes the proof of FP1

Proof of T.

We proved above that $\boldsymbol{\alpha}$ is locally Lipschitz continuous with respect to $V$ and we recall that $\widetilde{L}$ is $C^{1}$. Therefore $\widetilde{H}$ is also locally Lipschitz with respect to $V$. This and (A.3) implies that T holds.

Lemma A.3. Let $B, C \in \mathcal{M}_{2 \times 2}(\mathbb{R})$ be two positive definite matrices with eigenvalues $(1, r)$ and $(1, s)$ respectively, and $0<r \leq 1, s \geq 1$. Then for any $k>0$ the matrix $M$ defined by

$$
M=I_{d}+k(B C+C B)+k^{2} B C^{2} B,
$$

is positive definite with eigenvalues not smaller than 1.

Proof. We can assume that $B, C$ have the following form:

$$
C=\left(\begin{array}{ll}
1 & 0 \\
0 & s
\end{array}\right), \quad B=U\left(\begin{array}{ll}
1 & 0 \\
0 & r
\end{array}\right) U^{T}, \text { with } U \in \mathcal{O}_{2}(\mathbb{R}),
$$

since the eigenvalues of $M$ are invariant by taking the conjugate of $B$ and $C$ by the same orthogonal matrix. The same argument and noticing that $C$ commutes with $\left(\begin{array}{cc}1 & 0 \\ 0 & -1\end{array}\right)$, imply that we can assume that $U$ admits a positive determinant, and thus we can write it as

$$
U=\left(\begin{array}{cc}
\cos \chi & \sin \chi \\
-\sin \chi & \cos \chi
\end{array}\right)
$$

with $\chi \in[0,2 \pi)$. In this case, $M$ is given by

$$
\begin{aligned}
M & =I_{d}+k(B C+C B)+k^{2} B C^{2} B \\
& \sim I_{d}+k U^{T}\left(\begin{array}{ll}
1 & 0 \\
0 & s
\end{array}\right) U\left(\begin{array}{ll}
1 & 0 \\
0 & r
\end{array}\right)+k\left(\begin{array}{ll}
1 & 0 \\
0 & r
\end{array}\right) U^{T}\left(\begin{array}{ll}
1 & 0 \\
0 & s
\end{array}\right) U+k^{2}\left(\begin{array}{ll}
1 & 0 \\
0 & r
\end{array}\right) U^{T}\left(\begin{array}{cc}
1 & 0 \\
0 & s^{2}
\end{array}\right) U\left(\begin{array}{ll}
1 & 0 \\
0 & r
\end{array}\right) .
\end{aligned}
$$

We name $\widetilde{M}$ the matrix in the last line of the latter calculation, $M$ and $\widetilde{M}$ have the same eigenvalues. Let us compute $\widetilde{M}$

$$
\widetilde{M}=\left(\begin{array}{cc}
\cos ^{2} \chi(1+k)^{2}+\sin ^{2} \chi(1+k s)^{2} & -k(s-1)[1+r+k r(1+s)] \cos \chi \sin \chi \\
-k(s-1)[1+r+k r(1+s)] \cos \chi \sin \chi & \cos ^{2} \chi(1+k r s)^{2}+\sin ^{2} \chi(1+k r)^{2}
\end{array}\right),
$$

its trace is given by

$$
\operatorname{tr}(\widetilde{M})=\cos ^{2} \chi(1+k)^{2}+\sin ^{2} \chi(1+k r)^{2}+\cos ^{2} \chi(1+k r s)^{2}+\sin ^{2} \chi(1+k s)^{2},
$$

and its determinant by

$$
\begin{aligned}
\operatorname{det}(\widetilde{M})= & (1+k)^{2}(1+k r s)^{2} \cos ^{4} \chi+(1+k r)^{2}(1+k s)^{2} \sin ^{4} \chi \\
& +2(1+k)(1+k r)(1+k s)(1+k r s) \cos ^{2} \chi \sin ^{2} \chi \\
= & {\left[(1+k)(1+k r s) \cos ^{2} \chi+(1+k r)(1+k s) \sin ^{2} \chi\right]^{2} . }
\end{aligned}
$$

The eigenvalues of $\widetilde{M}$ are the roots of the following second-order polynomial function,

$$
X^{2}-\operatorname{tr}(\widetilde{M}) X+\operatorname{det}(\widetilde{M}),
$$


its smallest root is

$$
\frac{1}{2}\left(\operatorname{tr}(\widetilde{M})-\sqrt{\operatorname{tr}^{2}(\widetilde{M})-4 \operatorname{det}(\widetilde{M})}\right)
$$

which is not smaller than 1 if and only if

$$
\operatorname{tr}^{2}(\widetilde{M})-4 \operatorname{det}(\widetilde{M}) \leq(\operatorname{tr}(\widetilde{M})-2)^{2}
$$

Therefore, it is sufficient to check that $\operatorname{tr}(\widetilde{M}) \leq \operatorname{det}(\widetilde{M})+1$ to conclude. We define the function $f: \mathbb{R} \rightarrow \mathbb{R}$ by

$$
\begin{aligned}
f(x)=(1+k)^{2}(1+k r s)^{2} x^{2}+(1+k r)^{2}(1+k s)^{2}(1-x)^{2} & \\
+2(1+k)(1+k r)(1+k s)(1+k r s) x(1-x) \cos ^{2} \chi(1+k)^{2} & \\
& +\sin ^{2} \chi(1+k r)^{2}+\cos ^{2} \chi(1+k r s)^{2}+\sin ^{2} \chi(1+k s)^{2}+1 .
\end{aligned}
$$

This is a second-order polynomial in $x$ with

$$
\begin{aligned}
f(0) & =\left((1+k r)^{2}-1\right)\left((1+k s)^{2}-1\right) \geq 0 \\
f(1) & =\left((1+k)^{2}-1\right)\left((1+k r s)^{2}-1\right) \geq 0, \\
f^{\prime \prime}(x) & =2[(1+k)(1+k r s)-(1+k r)(1+k s)]^{2} .
\end{aligned}
$$

If $(1+k)(1+k r s)-(1+k r)(1+k s)=0$, then $f$ is linear and thus $f(x) \geq 0$ for all $x \in[0,1]$.

If $(1+k)(1+k r s)-(1+k r)(1+k s) \neq 0$, then the minimum of this polynomial function on $\mathbb{R}$ is obtained at $x_{\text {min }}$ defined as

$$
\begin{aligned}
x_{\min } & =\frac{(1+k)^{2}+(1+k r s)^{2}-(1+k s)^{2}-(1-k r)^{2}}{2[(1+k)(1+k r s)-(1+k r)(1+k s)]^{2}} \\
& =\frac{\left(1-r^{2}\right)\left(1-s^{2}\right) k^{2}+2(1-r)(1-s) k}{2[(1+k)(1+k r s)-(1+k r)(1+k s)]^{2}} \leq 0,
\end{aligned}
$$

since $0<r \leq 1, s \geq 1$ and $k>0$. Thus $f$ has no local minimum on $[0,1]$, then $f(x) \geq 0$ for all $x \in[0,1]$ since $f(0) \geq 0$ and $f(1) \geq 0$.

Since $\operatorname{det}(\widetilde{M})-\operatorname{tr}(\widetilde{M})+1=f\left(\cos ^{2} \chi\right) \geq 0$, this concludes the proof of the lemma. 